\title{
A Multi-Objective Optimization Approach to Optimal Sensor Location Problem in IGCC Power Plants
}

\author{
Authors \\ Pallabi Sen, Kinnar Sen, Urmila M. Diwekar* \\ Center for Uncertain Systems, Tools for Optimization and Management, Vishwamitra Research \\ Institute, Clarendon Hills, IL 60514, USA
}




\begin{abstract}
Integrated Gasification Combined Cycle (IGCC) power plants provide a cleaner and more efficient way to obtain energy from coal. In order to operate an IGCC power plant in a safe and stable manner, several input and output process parameters need to be monitored. However, due to economic and operational constraints, it is infeasible to place sensors at every input and output process parameter location. Hence, it becomes important to select the most effective sensor locations which lead to maximum information gain about the plant conditions. Practical issues present in an IGCC power plant, such as harsh physical conditions and variability in process parameters, make the optimal sensor location problem an especially complicated one. Further, sensors can have multiple objectives and they can produce uncertainties due to measurement errors. This work considers hybrid hardware and virtual sensing for advanced power systems with multiple objectives. In order to solve this real world large scale problem, we use a novel algorithm called Better Optimization of Nonlinear Uncertain Systems (BONUS). BONUS works in probability distribution space and avoids sampling for each optimization and derivative calculations iterations. A new algorithm for multi-objective optimization is also developed specifically for problem. The result of this nonlinear stochastic multi-objective problem is the non-dominated, or Pareto set, which provides trade-offs between various objectives like observability, cost, and thermal efficiency. This is the first attempt at the problem of optimal sensor deployment in advanced power plants, with consideration of hybrid hardware and virtual sensing and incorporation of uncertainty with multiple objectives.
\end{abstract}

\title{
1. INTRODUCTION
}

In an IGCG plant, it is crucial to have effective monitoring and control of various operational parameters for safe and profitable operation. Sensors play an essential role in process monitoring and control because they quantify parameters such as temperature, pressure, flow, and composition. A variety of available sensors range in cost, accuracy, and suitability for the operating conditions encountered in an IGCC plant. Certain regions in an IGCC plant operate under harsh environments, which may cause the sensors assigned to such locations to fail. If the sensors fail, they can damage not only process equipment, but also give rise to a number of issues related to plant operation. Sensor failures can be avoided by placing the sensors in a safer location that is not directly exposed to extreme conditions. But, this will result in a reduction of measurement accuracy. Virtual sensing using models is an alternative approach for locations where hardware sensors cannot be placed. So, the development of an optimized sensor network design, involving both hardware and virtual sensors, which specifies the location, number, and type of sensors will ensure better process control of the IGCC plant and is the focus of this work. 
The optimal sensor network design problem may be solved for the purpose of meeting certain objectives such as thermal efficiency, observability of parameters, emission level, increased plant life, effectiveness to detect equipment failure and reliability while expending the fewest possible resources. It can be easily inferred that the simultaneous achievement of more than one of such objectives may often be important for stakeholders. Therefore, a multi-objective optimization framework is necessary. Uncertainties are inherent in such a problem due sensor measurement errors. Hence, the problem of sensor placement is formulated as a multi-objective stochastic programming problem, the solution of which is the optimal sensor deployment policy for a specific advanced power system in the face of uncertainties in performance, efficiency, and power demand. In order to solve this large scale multi-objective optimization under uncertainty problem, a new algorithmic framework based on the Better Optimization of Nonlinear Uncertain Systems (BONUS) algorithm $(1,2)$ and a new multi-objective optimization algorithm developed specifically for this problem are used. This is the first attempt at the problem of optimal sensor deployment in advanced power plants with consideration of hybrid hardware and virtual sensing and with the incorporation of uncertainty with multiple objectives.

Advanced modeling techniques can be used to simulate the behavior of the IGCC plant with considerable accuracy (3-5). In this work, a simulation model of the IGCC plant, built using ASPEN Plus software, is used to evaluate the effects of the high levels of measurement uncertainty that exist within sensors subjected to harsh environments. After introducing variability into several process inputs of the simulation model, the resulting variance of the downstream variables (efficiency being one of them) were studied. This variability and sensor accuracy were then analyzed using techniques from information theory \& the BONUS algorithm (4), which determined which signals can be observed using virtual sensors, and which signals should be measured using a network of on-line sensors (i.e., sensors physically placed in the plant environment) to maximize observability. A similar approach was also used for maximizing plant thermal efficiency. Using a two-tier constraint method specific for this problem, both observability and efficiency the above objectives were maximized and cost of sensors was minimized. We generated a non-dominated surface for decision makers to select the appropriate design based upon their needs and budget.

The overall objective of this work is to develop innovative methodologies for optimal sensor deployment in advanced power plants and fuel systems with multiple objectives like observability, efficiency, and cost in the face of uncertainties. Specific contribution of this research are following: (1) developed a real time hybrid sensing framework with detailed computational models in ASPEN Plus and the new algorithmic framework, (2) designed a novel multi-objective stochastic programming framework for optimal sensor placement for optimal sensor placement under uncertainty, and (3) solved a real world sensor placement problem for IGCC systems sensor placement with multiple objectives (like observability, efficiency, and cost) while considering measurement uncertainties. 
The paper is divided into following sections. Sections 2 describes the literature survey followed by the IGCC plant sensor placement problem description. The BONUS algorithm is described in section 4 followed by the multi-objective optimization framework. Section 6 presents results and discussions and section 7 is the summary of work.

\section{LITERATURE SURVEY}

Optimal sensor placement problem has been addressed in the literature in the context of engineering structures (6-8), water security networks (9-11), traffic control (12), contamination detection (13), biomedical engineering (14), and chemical process industry $(15,31)$. Sensor placement for monitoring chemical processes is a fertile area of research, and a number of articles have been published in this area. Since this is very relevant to our work, we are presenting literature.

Relevant works include determining optimal sensor location in linear and nonlinear systems. Ali and Narasimhan (15) presented a method for determining the optimal location of sensors in a linear steady-state process by maximizing the reliability of the sensor measurements. Alonso et al.. (16) used a reduced order, linear dynamical model of a chemical reactor to determine the optimal sensor locations and sensor type by solving a min-max optimization problem. However, these approaches are applicable only to linear systems. More recently, researchers have looked at optimal sensor deployment for nonlinear systems. Singh and Hahn (17) developed a technique for sensor placement that could be applied to stable nonlinear systems such as binary distillation columns. D'Souza and Epureanu (18) developed an integrated approach for sensor placement and damage detection assessment that can be applied to both linear and nonlinear structures.

Different methodologies have been applied for solving optimal sensor placement problems. Some commonly used methods include optimality criteria based on the error covariance matrix (19-23) and observability matrix or Gramian $(18,24)$. Importance has been given to relevant aspects such as measurement cost, sensor failure, redundancy and process information (25-28). For example, Bagajewicz (29) and Chmielewski et al.. (30) present techniques related to the minimization of cost, subject to constraints related to data reconciliation, while Muske and Georgakis (31) present a sensor location technique that provides the best compromise between measurement cost and process information . Still,each of these are restricted to linear systems.

Considering the work specific to optimal sensor placement in IGCC plants, the following works can be cited. Sinumani et al. (32) developed a nonlinear programming problem for sensor deployment in an IGCC gasifier model, which they solved using an outer approximation based algorithm with the intention of minimizing sensor cost subject to the estimation accuracy of a sensor set. Lee and Diwekar (33) used Fisher information as a metric of observation in order to find the optimum location of sensors, subject to specified cost constraints, and applied BONUS algorithm to solve the stochastic nonlinear problem. 
Among sensor network design problems directly related to IGCC power plants, the work done by Paul et al.. (34) is noteworthy. They developed a Sensor Network Design (SND) algorithm to maximize plant efficiency of the Acid Gas Removal (AGR) section of the IGCC plant using dynamic programming in an estimator based control system and solved the resultant Mixed Integer Non-Linear Programming (MINLP) problem using a sequential approach of genetic algorithms and direct substitution methods. However, this method is shown to be computationally intensive.

The multi-objective optimization by solving sensor placement problem has been explored in various fields although there is no work at present specifically for IGCC power plants. Anpalagan et al. (35) have addressed the multi-objective optimization problem in design of wireless sensor networks. In their work, they discuss multiple algorithms to solve optimization problems such as GA, Particle Swarm Optimization, metaheuristic algorithms etc. Stehlik et al. (36) demonstrated that multi-objective problems can provide a more accurate detection of intrusion in wireless sensor networks than single objective problems can. Aral et al. (37) used multi-objective optimization for sensor placement in water distribution systems. They used the sub-domain concept to overcome the difficulty of finding optimal sensor locations from thousands of candidate locations within vast water distribution systems.

\section{IGCC SYSTEM SENSOR PLACEMENT PROBLEM}

The Integrated Gasification Combined Cycle (IGCC) technology integrates coal gasification and power generation processes so that they can be carried out in the same plant. Normally, combined cycle plants utilize the waste heat from the gas turbine section to produce steam for the steam turbines. However, the IGCC technology further improves efficiency by salvaging the steam produced during gasification and sending it to the steam turbines for power generation.

The IGCC process flow (figure 1) consists of three main blocks - the Air Separation Unit (ASU), the gasifier, and the power generation unit. This study is based on the Polk Power Station owned by Tampa electric company in Florida. $(38,39)$ 
Figure 1 - A simplified process flow diagram of the IGCC process plant. (Source: (33))

The main purpose of the ASU is to provide the oxygen required to support the high temperature reactions carried out in the gasifier. The nitrogen produced in the ASU is sent to the gas turbine to reduce NOx generation. The ASU produces $22.05 \mathrm{~kg} / \mathrm{second}$ of oxygen at $96 \%$ purity and $63 \mathrm{~kg} / \mathrm{second}$ of nitrogen at $98.5 \%$ purity.

The Tampa power plant uses a GE entrained flow slagging gasifier which was originally developed by Texaco. This entrained flow slagging gasifier operates at high temperatures (the ash remains in liquid slag form, hence the term "slagging") \& pressures of around $1645 \mathrm{~K}$ and $2760 \mathrm{KPa}$. Due to such high temperatures, high carbon conversion rates can be achieved. As a result, this type of gasifier is capable of handling a larger throughput.

The coal feed is first broken down in rod mills and then mixed with water to form a slurry which is then fed to the gasifier. Three main reactions take place during gasification. The first is pyrolysis, or coal de-volatilization, where the coal feed is thermally decomposed to produce volatiles $\mathrm{CO}_{2}, \mathrm{CO}, \mathrm{CH}_{4}$ and char. Next is combustion where char and some volatiles react with oxygen to mainly produce $\mathrm{CO}_{2}$, as well as some $\mathrm{CO}$. Finally, gasification takes place when steam and air are introduced in the ratio of approximately 3:1 to react with char to produce $\mathrm{CO}_{2}$. At the same time, the reversible water-gas shift reaction occurs and converts $\mathrm{CO}$ into $\mathrm{CO}_{2}$.

The final mixture of gases leaving the gasifier, known as syngas, consists mainly of hydrogen $\left(\mathrm{H}_{2}\right)$, water vapor $\left(\mathrm{H}_{2} \mathrm{O}\right)$, carbon monoxide $(\mathrm{CO})$, and carbon dioxide $\left(\mathrm{CO}_{2}\right)$, while smaller amounts of hydrogen sulfide $\left(\mathrm{H}_{2} \mathrm{~S}\right)$, carbonyl sulfide (COS), methane $\left(\mathrm{CH}_{4}\right)$, argon (Ar), nitrogen $\left(\mathrm{N}_{2}\right)$, and ammonia $\left(\mathrm{NH}_{3}\right)$. Besides syngas, which is the desired product, slag and flyash are also formed in the gasifier as by-products. The syngas is subsequently water scrubbed to remove flyash and other particulate matter.

The power generation section consists of the gas combustion turbine, the heat recovery section, and the steam turbines. Clean syngas from the gasification section is introduced into the individual combustion cans of the combustion turbine from the fuel skid through a ring header. Nitrogen from the ASU is also introduced in a similar fashion into the combustion cans to reduce NOx emission. Air required for combustion is provided by a powerful multi-stage compressor. The exhaust leaves the gas turbine at a temperature of 
around $570^{\circ} \mathrm{C}$ and goes to Heat Recovery Steam Generators (HRSG). The heat from the exhaust is used to produce or re-heat steam in the various sections of the HRSG. Thus, the IGCC power plant model is a large, complex system with more than 200 streams and 80 unit operations with a large number of design specifications.

In order to ensure that the plant operates in a safe, reliable and profitable manner, efficient process monitoring and control is of utmost importance. This is why we need a sensor network to gain real time information about the hundreds of process variables in the plant. The most obvious benefit of having a sensor network is an increase in the observability of plant parameters. This is important for maintaining all process variables within a safe range of operation at all times.

Another benefit is increased plant efficiency. Certain key process parameters should always be maintained as close to their target value as possible because they affect the efficiency of the plant. One such parameter is gasifier temperature. A higher than optimal temperature decreases the durability of the gasifier itself (it increases the capital and operating expenditures), while lower temperatures increase the formation of slag (lower carbon conversion rate). Since power plants are designed for maximum efficiency, any deviation in the process variables causes the plant to operate at suboptimal condition. Sensors are capable of increasing the prediction accuracy of such variables by reducing the errors in measurement. This in turn helps in taking appropriate corrective action to adjust the value of the concerned process variable and bring it closer to its optimal value.

Nevertheless, for an intricate system like the IGCC plant, with harsh operating conditions and hundreds of process variables that need to be mapped in real time, it is physically and economically infeasible to install sensors for monitoring each and every variable. This is why virtual sensing or soft sensing is a great advantage for process monitoring and controlling in large, complex systems such as IGCC plants by providing a much more economical and less-invasive alternative to gather information.

Virtual sensing is the process by which an estimated value of a variable is calculated through mathematical modeling without placing any direct physical means of measurement such as a sensor (40). Estimates are made with the help of analytical methods, such as mathematical or computational modeling, which are based on either physical laws that define the system operation or empirical methods based on historical data of either that particular variable or other variables to which it is related.

By eliminating the need to place actual sensors, virtual sensors help reduce capital costs as well as maintenance costs. Moreover, it is not possible to place sensors in hostile areas of the IGCC plant because of the very high risk of damage. For example, gasifier temperature, an important process variable, is difficult to measure directly because thermocouples are damaged rapidly when inserted into the hot face of the gasifier. However, it is known that the methane production rate in the last stage of the gasifier is dependent upon the gasifier temperature and fuel composition. So, by collecting information about methane production rate and fuel composition (both parameters can be 
easily measured physically), it is possible to infer the gasifier temperature, and thus have a virtual sensor for methane.

The virtual sensing method employed in this project is based on a simulation model of the IGCC plant developed using the ASPEN Plus software package. ASPEN Plus is one of the leading chemical process optimization software(s) currently used throughout the industry to model and simulate chemical process plants. This software is capable of performing complex and tedious calculations which use the laws of thermodynamics, chemical reaction kinetics, mass and heat transfer etc. to predict the properties of output streams once information about input streams and equipment specifications have been provided.

Although virtual sensing has many advantages, it does have one major disadvantage that cannot be overlooked. Estimating the value of a process variable through virtual sensing creates uncertainty in measurement. This measurement error is much higher (around \pm $20 \%$ ) than that from real sensors. Depending upon the accuracy of sensors (directly proportional to cost), the measurement error can reduce to $\pm 5 \%$ for low accuracy sensors, $\pm 2.5 \%$ for medium accuracy sensors and $\pm 1 \%$ for high accuracy sensors. Due to these errors, we cannot use virtual sensing indiscriminately. Hence a hybrid sensor network design that employs both real and virtual sensors needs to be developed.

This present optimal sensor network design was developed with three objectives in perspective- to maximize the observability of plant parameters (measured in terms of Fisher Information), to maximize the thermal efficiency of the plant, and to minimize the cost of purchasing the sensors. 
Table I - List of 24 intermediate variables. (Source: (33))

The candidate locations where sensors can be installed in the plant and the type of sensors (low, medium or high accuracy/cost) both are the decision variables. There a total of 24 candidate locations, where the sensors can be installed (presented in Table I). These locations correspond to 24 variables which will henceforth be referred to as the "intermediate" variables. These variables have been chosen because they influence the important output parameters of the plant such as plant power production or gasifier performance (based upon knowledge of the IGCC process) which need to be maintained at their target levels in order for the plant to operate optimally. Figure 2 depicts the intermediate locations in the plant represented by the letter " $y$ " followed by a subscript. The higher the subscript, the further downstream is the variable located. The measurement error of any process variable is influenced by all other variables upstream of it and this fact needs to be accounted for in the problem formulation.

Figure 2 - Potential sensor locations in the IGCC power plant (Source: (33)) 
The sensor network design problem is further complicated by the following factors. The presence of uncertainty in the process variables due to system and measurement noise makes the problem stochastic in nature. Moreover, the equations governing the physical processes taking place in the IGCC power plant are nonlinear which in turn makes our optimization problem nonlinear. The multitude of process variables and the intricate relationships between them make it difficult to pick which variables should be measured directly and which ones should be measured through virtual sensing. Since certain decision variables can take only integer values, this problem is a mixed integer nonlinear programming problem. Finally, since the problem attempts to simultaneously address the optimization of observability, thermal efficiency and cost, it becomes a multi-objective optimization problem. In summation, the optimal sensor network design problem described in this paper is a stochastic mixed integer nonlinear multi-objective problem.

\subsection{Fisher Information Maximization}

The behavior of the process system can be captured through model, where uncertainty may be prevalent in the system and reflected in the measurement dynamics. The Kalman Filter (KF) is well-developed sequential algorithm for estimating the variability of the state variables for a linear system (41), whereas the Extended Kalman Filter (EKF) addresses processes that involve nonlinear dynamics. The EKF is a suboptimal variation of the KF that uses a linearization of the system equations around the nominal operating point. Estimation is carried out recursively and the posterior covariance matrix is generated for observability calculation.

Observability is measured by normalizing the covariance, of estimated process variables,

$\mathrm{Pk}$, by the covariance from the corresponding on-line sensor, $O_{k}=\sqrt{P_{k} / P^{s}}$ (where $P^{s}$ be the state covariance if each sensor is placed on-line). Values of $O_{k}$ close to 0 (1) indicate the best (worst) index of observability. The observability indexes are then ranked using a Pareto analysis, and the optimal sensor network is chosen by selecting the set of sensors that provide the best measure of observability for estimating the state of the dynamical system.

For the purpose of comparing to the Fisher information method presented in the following subsection, it is worth noting that through the canonical parameterization, $\wedge \mathrm{k}=$ $\mathrm{Pk}-1$, and $\mathrm{Lk}=\mathrm{Pk}-1 \mid \mathrm{k}$, the dual to the KF, also known as the Information Filter (IF), can be derived with an equivalent observability index $O_{k}=\sqrt{\Omega^{s} / \Omega_{k}}$. It follows that the observability index based on KF is a special case of Fisher information matrix in which the uncertainty is represented by normal distribution. However, the limitations of the KF or EKF analysis lies in the following assumptions that are made in order to find the observability of a particular network: (1) It employs a reduced order dynamic model based on linearization of the highly nonlinear model of IGCC system, which may not be 
appropriate for all operating conditions; (2) All disturbances and errors are assumed to be normally distributed, which may not be the case in practice. In order to relax these assumptions, we use a novel approach based on the Fisher information described below.

Fisher information (FI), named after Ronald Fisher (42), is commonly used in information theory as a measure of the information contained by a random, observable variable $X$ about $\Theta$, an unknown parameter of its own probability distribution function. In the SND problem, FI is a measure of the information contained by a set of independent and identically distributed observations, $\mathrm{x}_{1}, \mathrm{x}_{2}, \ldots, \mathrm{x}_{\mathrm{n}}$ of a process variable about its nominal value.

FI captures the amount of information that the observations, $\mathrm{x}_{1}, \mathrm{x}_{2}, \ldots, \mathrm{x}_{\mathrm{n}}$, contain about some unknown parameter, $\theta_{\mathrm{x}}$, within its own distribution (e.g., mean or variance), $\mathrm{p}_{\mathrm{x}}(\mathrm{x})$, by quantifying the expected change in the distribution due to a change in this parameter value. Thus, we can say that FI is a statistical measure of dynamic system order that quantifies the variability in a process variable as a function of changes in the mean value of the process variable. Therefore, the following mathematical expression for $\mathrm{I}_{\mathrm{x}}\left(\theta_{\mathrm{x}}\right)$ can be used for FI, . (43)

$$
I_{x}\left(\theta_{x}\right)=E^{x}\left[\left(\frac{1}{p_{x} \mid\left(x \mid \theta_{x}\right)} \frac{\partial p_{x} \mid\left(x \mid \theta_{x}\right)}{\partial \theta_{x}}\right)^{2}\right]
$$

The distribution $\mathrm{p}\left(\mathrm{x} \mid \theta_{\mathrm{x}}\right)$ is the likelihood of $\mathrm{x}$, given the parameter $\theta_{\mathrm{x}}$. There are various other statistical measures similar to FI such as Shannon information, Boltzmann-Gibbs, Tsallis, Renyi and Sharma- Mittal entropies, which can also be used to capture the amount of information that a set of observations contains about its own distribution properties (44). But, FI was chosen as the suitable metric for observability in the sensor placement problem primarily because of its local property.

To appreciate the importance of FI's local property, it should be noted here that the true value of an input process variable, for example, coal slurry feed rate, could change with time. This would produce a corresponding shift in the sensor measurement distribution. The likelihood functions of all the process variables satisfy the shift-invariant property. By using the gradient operator, $\partial / \partial \Theta x$, FI measures the amount of change in the likelihood function due to a change in the unknown parameter value. Thus, the values of FI are directly proportional to the rate of change of the likelihood function with respect to a change in the parameter value, and consequently, to the level of observability in estimating the true parameter value.

One of our objectives is to design an optimal network of sensors such that the variability in the estimations of unmeasured process variables can be minimized. Within the scope of this work, FI is viewed as a statistical measure of system order. Hence, maximizing FI will result in better observability. To do so, the following nonlinear, stochastic mixed- 
integer programming problem is formulated where the objective is to maximize the overall FI, subject to the costs associated with placing a network of on-line sensors.

$$
\begin{array}{r}
\max _{W_{j \tau}} \sum_{\tau=1}^{3} \quad \sum_{j=1}^{S_{\text {out }}} f_{j \tau}\left(W_{j \tau}, Y_{j}\right) \\
\text { Subject to } \quad \sum_{\tau=1}^{3} \quad \sum_{j=1}^{S_{\text {out }}} C_{j \tau} W_{j \tau} \leq B \\
\sum_{\tau=1}^{3} W_{j \tau} \leq 1, \quad j=1,2, \ldots S_{\text {out }}
\end{array}
$$

Where $W_{j \tau} \in(0,1)$ and $\mathrm{j}=1,2, \ldots \mathrm{S}_{\text {out }}, \tau=1,2,3$

Here, the objective function is expressed as the sum of $f_{j}$ terms for all the 24 locations. The objective function $\mathrm{f}_{\mathrm{j}}$ is a function of Wand $\mathrm{Y}$, where, $\mathrm{W}$ is the binary variable which takes on the value 1 if a sensor is placed at location $\mathrm{j}$ or the value of 0 if it is not placed. $\tau$ is an integer variable that indicates the type of sensor used (can take the values 1,2 or 3 indicating low cost, medium cost and high cost sensors respectively) and $\mathrm{Y}$ is a random variable associated with the measurement uncertainties in the intermediate process variables. The objective function should be maximized to satisfy the cost constraint where $C_{j}$ represents the cost of placing a sensor at location $\mathrm{j}$ and $\mathrm{B}$ represents the available budget.

The objective term, $f_{j \tau}(W, Y)$, was calculated as follows. It is assumed that the information collected about the distribution properties of a process variable is always greater if a sensor is a real sensor placed on-line at a specific location or at any location upstream of it. This assumption is valid because the information gained through actual sensing is always greater than the information gained through virtual sensing. If this were not the case, placing real sensors would provide no additional information at that location and hence would not be desirable at all. So, moving forward with this assumption, $\mathrm{I}^{\mathrm{S}}$ $\left(\theta y_{j} \mid w_{k}=1\right)$ is defined at the FI about parameter $\theta y_{j}$ corresponding to process variable $\mathrm{j}$ resulting from a sensor placed at location $\mathrm{k}=1,2, \ldots$, and $\mathrm{I}^{\mathrm{ns}}\left(\theta \mathrm{y}_{\mathrm{j}} \mid \mathrm{w}_{\mathrm{k}}=1\right)$, where $\mathrm{k}=1$, $2, \ldots, 24$, is defined as the FI of $\theta y_{j}$ resulting from no sensors placed in the network of intermediate and output variables such that $\mathrm{I}^{\mathrm{s}}\left(\theta \mathrm{y}_{\mathrm{j}} \mid \mathrm{w}_{\mathrm{k}}=1\right) \geq \mathrm{I}^{\mathrm{ns}}\left(\theta \mathrm{y}_{\mathrm{j}}\right), \mathrm{j}=1,2, \ldots 24$. Suppose a sensor is placed at location $\mathrm{k}$ that is upstream of variable $\mathrm{j}$. The information gained from placing the sensor at location $\mathrm{k}$ can also increase the amount of information about the variable at location $\mathrm{j}$. Hence the function $f_{j \tau}(\mathrm{w}, \mathrm{Y})$ is be defined as:

$$
f_{j}(w, Y)=\sum_{k=1}^{S_{\text {out }}}\left(1-I^{n s}\left(\theta_{Y_{j}}\right)\right) / I^{s}\left(\theta_{Y_{j}} \mid w_{k}=1\right)
$$

This equation captures the overall effect of placing a sensor on all other process variables by summing the resulting information gain from placing a sensor at location $\mathrm{k}$ across the entire set of intermediate and output process variables. 


\subsection{Efficiency Maximization}

The term efficiency can be used to mean more than one thing in case of an IGCC power plant. However, this work is focused on maximizing the plant thermal efficiency, which can be defined in the simplest terms as the ratio of the net power generated in the IGCC plant and the chemical energy contained in the coal feed. The thermal efficiency has been calculated based on the HHV (Higher Heating Value) of coal.

One expression that can represent the above definition is as follows:

$$
E=\frac{P_{\text {wrnet }}}{F_{\text {coalc }} \times F_{m f} \times H O C}
$$

The term "Pwrnet", measured in Watts, represents the net power generated. This is obtained by subtracting the miscellaneous power consumed due to the plant operation from the sum of the power generated in the plant. " $\mathrm{F}_{\text {coalc }}$ ' is the feed rate of coal slurry into the gasifier measured in $\mathrm{kg} / \mathrm{s}$. " $\mathrm{Fmf}$ " is the weight \% of coal in dry basis (without considering moisture) in the coal feed. "HOC" represents the heat of combustion of coal expressed in $\mathrm{J} / \mathrm{kg}$.

The objective function for expected value of efficiency maximization is a nonlinear, stochastic, mixed integer problem which is of the similar form as that of FI.

$$
\begin{gathered}
\max _{W_{j \tau}} \sum_{\tau=1}^{3} \quad \sum_{j=1}^{S_{\text {out }}} E\left[\eta_{j \tau}\left(W_{j \tau}, Y_{j}\right)\right] \\
\text { Subject to } \quad \sum_{\tau=1}^{3} \quad \sum_{j=1}^{S_{\text {out }}} C_{j \tau} W_{j \tau} \leq B \\
\sum_{\tau=1}^{3} W_{j \tau} \leq 1, \quad j=1,2, \ldots S_{\text {out }}
\end{gathered}
$$

Here, E refers to expected value of the objective function. The objective function is expressed as the sum of $\eta_{j}$ terms for all the 24 locations. $\eta_{j}$ defined below in equation 10 is a function of the increase in efficiency for placing a sensor at location $\mathrm{j}$. If sensors are placed at none of the 24 intermediate variable locations and all of them are measured through virtual sensing, then the plant thermal efficiency is only $36.14 \%$ due to high measurement uncertainties. As sensors are placed at these locations, the variability in the measurement of the intermediate variables reduces and this leads to an increase in efficiency. This is why $\eta_{\mathrm{j}, \tau}$, the incremental change in efficiency, is defined in the following manner (similar to the expression used for FI maximization). It is designed to assume greater values for locations where placing a sensor produces the largest incremental change in efficiency. 


$$
\eta_{j}(w, Y)=\sum_{k=1}^{S_{\text {out }}}\left(1-E^{n s}\left(\theta_{Y_{j}}\right)\right) / E^{s}\left(\theta_{Y_{j}} \mid w_{k}=1\right)
$$

Here, $E^{\text {ns }}$ and $E$, are the values of plant thermal efficiency obtained before and after placing a sensor at location $\mathrm{j}$ respectively. For the highest available budget, the efficiency was calculated to be $44.78 \%$.

\section{BONUS ALGORITHM}

A generalized numerical optimization framework for solving deterministic optimization problem is shown in figure 3 . In general, the nonlinear numerical optimization procedure is iterative, where the values of the objective functions and constraints are calculated again and again for various feasible values of the decision variables by running the model until the optimal value of the objective function is found.

Figure 3 - Pictorial representation of the numerical optimization framework (Source(2))

In the case of the sensor deployment problem, the optimization algorithm would call upon the computational model of the process with an input set of values for the decision variables. The model would then run the simulation and calculate the values of the objective functions and constraints and send them back to the optimizer. Based upon this 
information, the optimizer would select a new set of values for the decision variables and this process is repeated until the optimal value of objective functions that satisfy the pertinent constraints are found.

The above mentioned methodology is sufficient for solving deterministic optimization problems. However, the sensor network design problem is stochastic in nature. Therefore, the optimization loop in figure 3 has to operate in conjunction with the sampling loop. In stochastic programming, the objective function has to be expressed in the form of a probabilistic function such as expected value, variance or fractiles. Hence, a probabilistic model has to be considered in the above figure instead of a deterministic one. The modified optimization framework has been shown in figure 4 where the deterministic model has been replaced by an iterative stochastic with a sampling loop.

Figure 4 - Pictorial representation of the stochastic programming framework (Source: (2))

The method depicted above is expected to be computationally intensive since the model has to be run repeatedly for each sample of the uncertain variables before the model can generate the required information for the optimization loop. 
Efficient sampling techniques as such can be employed to alleviate this issue to a certain extent. But a much more effective solution to this issue is provided by the BONUS (Better Optimization for Nonlinear Uncertain Systems) algorithm which is based on a reweighting scheme, a form of Reduced Order Model (ROM) in probability space. For more details of this algorithm, please refer to Diwekar and David (2).

The BONUS algorithm is based upon the reweighting approach that proposed by Sahin and Diwekar (1) and it eliminates the need of running the entire computational model for over and over again for subsequent iterations.

\subsection{Reweighting Approach to calculate Fisher Information \& Efficiency}

Using the ASPEN Plus environment, a comprehensive model of the IGCC process is used to simulate the steady-state performance of the ASU, gasifier, and power generation operations. This nonlinear ASPEN model is used to estimate the set of unmeasured variables using the data acquired from the process variables directly measured through the network of sensors physically deployed within the plant. Internal operating conditions in the process can be analyzed by varying a set of input variables, including coal and oxygen flow rate. These input variable operating conditions uniformly span a sample space surrounding their nominal values (45). A set of $N_{s}$ input variable operating conditions are generated using Hammersley sequencing, a low-discrepancy sampling method, to generate the uniform sample space. This sampling method is chosen over common Monte-Carlo sampling techniques because Hammersley sequencing provides a more uniform distribution across an d-dimensional sample space and the number of sample points necessary to sufficiently cover an d-dimensional space can be significantly reduced by using the Hammersley sampling technique $(45,46)$. Then, the IGCC process is simulated $N_{s}$ times, once per input operating condition, to generate a corresponding vector of points for Sout intermediate and output process variables, including the syngas temperature, pressure, and mass flow rate, among others. The resulting vector set of intermediate and output variables can be used to capture the nonlinear effects of the IGCC process, as well as the variability of downstream variables resulting from a uniformly distributed set of input variable sample points. The IGCC process is simulated across a space of operating points to generate the probability distribution of each intermediate and output variable due to variations in the input variables and the nonlinear process behavior. From this, the information about each of the process variables can be obtained as a result of a shift in the true steady-state operating point. In this work, we have used 800 sample runs of the IGCC model for the first iteration. However, running the model once even for 800 samples of this base distribution using the IGCC model takes around 18 hours, therefore, it is not feasible to create a new sample set of inputs and run the simulation to generate the sample space of the outputs over and over again for each iteration as shown in Figure 4. This is where BONUS eliminates the necessity to recreate a new sample space through simulation of the process behavior. It uses the following reweighting approach to reduce the computational intensity by more than $99 \%$. 
Figure 5 illustrates the nature of reweighting approach. On the first iteration, a set of $N_{s}$ sample points uniformly distributed across a d-dimensional sample space are used to perform $N_{s}$ simulation replications of IGCC process. Let $f_{0}\left(x_{i}\right)$ be the probability density function (PDF) associated with the base input distribution for input variables $x_{i}, i=1,2, \ldots S_{\text {in }}$ respectively. Following the simulation of the IGCC process at iteration $\mathrm{t}=0$, let $f_{0}\left(y_{i}\right)$ and $F\left(y_{i}\right)$ be the base PDF and cumulative distribution function $(\mathrm{CDF})$ associated with the intermediate and output variables $y_{j}, j=1,2, \ldots S_{\text {out }}$, where $y_{j}=h\left(x_{i}, i=1,2, \ldots S_{i n}\right), j=1,2, \ldots S_{\text {out }}$ is the nonlinear transformation from each input variable, $x_{i}$ to the downstream variable $y_{j}$.

Figure 5 - Pictorial representation of reweighting in BONUS algorithm (Source: (2))

Next, consider when a new input distribution is defined, such as when a sensor is placed at the location of an input variable. The redefined distribution $f_{t}\left(x_{i}\right)$, at iteration $t$ is used to create a set of weights

$$
W_{t}\left(x_{i}\right)=\frac{f_{t}\left(x_{i}\right)}{f_{0}\left(x_{i}\right)}, i=1,2, \ldots S_{\text {in }}
$$

This gives the likelihood ratio between the redefined and base distributions. Given the input variables act independently, these weights are used to construct the resulting distribution for the downstream intermediate or output variables at iteration $\mathrm{t}$ by multiplying the associated weights $W_{t}\left(x_{i}\right)$ with the base distribution $f_{0}\left(y_{j}\right)$,

$$
f_{t}\left(y_{j}\right)=f_{0}\left(y_{j}\right) \prod_{i=1}^{S_{i n}}\left(1+\gamma_{i j}\left(W_{t}\left(x_{i}\right)+1\right), j=1,2, \ldots, S_{\text {out }}\right.
$$

where $\gamma_{i j}=1(0)$ if variable $y_{j}$ is (is not) downstream of $x_{i}$. The distribution $f_{t}\left(y_{j}\right)$ is normalized using 
$\check{f}_{t}\left(y_{j}\right)=\frac{f_{t}\left(y_{j}\right)}{\sum_{I=1}^{N s} f_{t(n)}\left(\left(y_{j}(n+1)-y_{j}(n-1)\right) / 2\right.}$

This re-weighting approach can also be used when a sensor is placed at the location of an intermediate process variable, to construct the resulting change in distributions of corresponding downstream variables.

FI is defined in terms of derivative of density function. It can be calculated from Equation 13 as follows. Assume that the shift-invariant property holds for a small $\varepsilon \geq 0$ change in parameter $\theta_{y}$ (i.e. the mean value). This is a viable assumption around the mean operating conditions in an IGCC process, since it is desirable to operate the plant within a stable region. The density functions $\check{f}_{t}\left(y_{n}+\varepsilon\right)$ and $\check{f}_{t}\left(y_{n}-\varepsilon\right)$ are then calculated and used to generate an approximation of the first order derivative $\partial \check{f}_{t}\left(y_{n}\right) / \partial \theta_{y}$ given by

$$
\partial \check{f}_{t}\left(y_{n}\right) / \partial \theta_{y}=\frac{\left[\check{f}_{t}\left(y_{n}+\varepsilon\right)-\check{f}_{t}\left(y_{n}-\varepsilon\right)\right]}{2 \varepsilon}
$$

The FI is then obtained by substituting (14) into the discrete approximation of (1) to yield

$$
I_{y}\left(\theta_{y}\right)=\sum_{n=1}^{N s}(y(n)-y(n-1))\left[\partial \widetilde{f}_{t}\left(y_{n}\right) / \partial \theta_{y}\right]^{2} / \check{f}_{t}\left(y_{n}\right)
$$

Since efficiency is one of the output variables say $y_{m}$, the expected value of efficiency can be calculated using equation 16 as follows

$$
E\left(y_{m}\right)=\sum_{n=1}^{N s} \check{f}_{t}\left(y_{m}(n)\right) y_{m}(n)
$$

\section{MULTI-OBJECTIVE OPTIMIZATION FRAMEWORK}

The solution methodology used in this paper is based upon constraint methods where the basic strategy is to pick one of the objectives to minimize (say $\mathrm{Z}_{1}$ ), while each of the other objectives $(\mathrm{Zi}, \mathrm{i}=1, \ldots, \mathrm{k}, \mathrm{i} \neq 1)$ are turned into inequality constraints with parametric right-hand sides $(\varepsilon i, i=1, \ldots, k, i \neq 1)$. The problem takes the following form. 


$$
\text { Optimize } \quad Z_{\text {mult }}=Z_{i}
$$

subject to

$$
\begin{aligned}
& \text { For Minimization } Z_{j} \leq \varepsilon_{j}, j=1,2, \ldots, k ; j \neq i \\
& \text { For Maximization } \begin{aligned}
Z_{j} \geq \varepsilon_{j}, j & =1,2, \ldots, k ; j \neq i \\
h(x) & =0 \\
g(x) & \leq 0
\end{aligned}
\end{aligned}
$$

The single objective problems are solved repeatedly for different values of $\varepsilon_{\mathrm{i}}$ between the upper and lower bounds of the objective functions. This creates the Pareto set.

The first step in the constraint method is to find Pay-off tables, i.e. bounds for the objective functions. This is carried out by solving single objective optimization problems with only the original set of constraints. These bounds, $\varepsilon$, will form the RHS when all but one of the objective functions are converted into constraints. In order to efficiently find the Pareto set for our problems without solving a large number of infeasible optimization problems, we designed a two tier constraint method. We are aware that for a specific cost constraint we have an upper limit on efficiency beyond which value the problem will be infeasible. Therefore, instead of finding a single pay-off table by optimizing each objective separately, we first find the bounds on cost.

These bounds on the cost are $\mathrm{C}_{\min }$ and $\mathrm{C}_{\max }$, where $\mathrm{C}_{\min }$ is the cost of installing no sensors and $\mathrm{C}_{\max }$ is the cost of installing high accuracy sensors at all 24 locations. Eleven evenly spaced cost points were considered between $\mathrm{C}_{\min }$ and $\mathrm{C}_{\max }$ to obtain value of $\varepsilon_{\mathrm{k}}$ for the cost constraint.

Thereafter, the following two sets of problems are solved for each value of $\varepsilon_{\mathrm{k}}$ to now find bounds for FI and efficiency.

1. Maximizing FI

$$
\begin{gathered}
\max _{W_{j \tau}} \sum_{\tau=1}^{3} \quad \sum_{j=1}^{S_{o u t}} f_{j \tau}\left(W_{j \tau}, Y_{j}\right) \\
\text { Subject to } \quad \sum_{\tau=1}^{3} \quad \sum_{j=1}^{S_{\text {out }}} C_{j \tau} W_{j \tau} \leq \varepsilon_{k} \\
\sum_{\tau=1}^{3} W_{j \tau} \leq 1, \quad j=1,2, \ldots S_{\text {out }}
\end{gathered}
$$

2. Maximizing efficiency

$$
\text { 3. } \max _{W_{j \tau}} \sum_{\tau=1}^{3} \sum_{j=1}^{S_{\text {out }}} E\left[\eta_{j \tau}\left(W_{j \tau}, Y_{j}\right)\right]
$$




$$
\begin{gathered}
\text { Subject to } \quad \sum_{\tau=1}^{3} \quad \sum_{j=1}^{S_{\text {out }}} C_{j \tau} W_{j \tau} \leq \varepsilon_{k}, \\
\sum_{\tau=1}^{3} W_{j \tau} \leq 1, \quad j=1,2, \ldots S_{\text {out }}
\end{gathered}
$$

where $\mathrm{k}=1,2, . .10$

For the first set of problems where FI is maximized, the corresponding value of efficiency is noted. Similarly, in the second set of problems, we determined the sensor locations for maximum FI (observability), given the cost and calculated the corresponding efficiency. These values of FI and efficiency form the pay-off table for each value of $\epsilon_{k}$.

Table II: Optimal sensor network design for maximizing efficiency

Table II shows the solutions of the first set of problems discussed above where efficiency was maximized. There a total of 11 solutions considered. For each given budget constraint, the optimal locations for placing sensors is shown in the central columns of the table. The second and third columns from the left show the budget and FI obtained for this sensor network design while the column on the extreme right gives the maximum thermal efficiency. These values of FI provide lower bound values for FI corresponding to each cost constraint. 
Table III: Sensor network design for maximizing Fisher Information

Table III above shows the solutions for the second set of problems where FI has been maximized. As before, with increasing budget, as the number of sensors increases, their accuracy improves. It should be noted here that the maximum possible efficiency can be attained by using comparatively fewer number of sensors. This provides lower bound of efficiency values for the multi-objective constraint method problem formulation corresponding to each cost constraint.

From the above two tables, we can find the upper and lower bounds of FI and efficiency for each cost constraint and thereby generate 11 pay-off tables corresponding to each cost.

We then considered FI as the main objective function and solved the following single objective problem by changing the bounds $\varepsilon \varepsilon_{\mathrm{k}}$ of efficiency and $\mathrm{Bj}$ of cost.

$$
\max _{W_{j \tau}} \sum_{\tau=1}^{3} \quad \sum_{j=1}^{S_{o u t}} f_{j \tau}\left(W_{j \tau}, Y_{j}\right)
$$

$$
\begin{gathered}
\text { Subject to } \sum_{\tau=1}^{3} \quad \sum_{j=1}^{S_{o u t}} E\left[\eta_{j \tau}\left(W_{j \tau}, Y_{j}\right)\right] \geq \varepsilon \varepsilon_{k}, \\
\sum_{\tau=1}^{3} \quad \sum_{j=1}^{S_{o u t}} C_{j \tau} W_{j \tau} \leq B_{j j} \\
\sum_{\tau=1}^{3} W_{j \tau} \leq 1, \quad j=1,2, \ldots S_{\text {out }}
\end{gathered}
$$

where $\mathrm{jj}=1,10$ and $\mathrm{k}=1,10$. 
Here, $\varepsilon \varepsilon_{k}$ represents the lower bound on efficiency and $\mathrm{B}_{\mathrm{j}}$ represents the upper bound of cost in bin number " $\mathrm{j}$ ". In order to generate more points on the Pareto Surface, this problem was solved many more times by creating additional bounds on efficiency in increments of $0.5 \%$ i.e., additional constraints were created with new values of $\varepsilon \varepsilon_{k}$ that increased by $0.5 \%$ each time. There are 110 single optimization problems to be solved in this manner corresponding to the 11 ten cost bins and 10 values of efficiency. We solved this problem considering only high accuracy sensors at first and then subsequently by considering all three types, i.e., high-medium-low accuracy sensors, together as we became aware of the non-convexities of the problem. The analysis of the Pareto surfaces thus generated has been carried out in the results \& discussion section. Although, in this work we have used FI as the objective and efficiency as a constraint, we could have constrained FI and considered efficiency as the objective without changing the results.

\section{RESULTS AND DISCUSSIONS}

In order to analyze the Pareto points generated so far, they were plotted in a series of 3-D graphs. These graphs provide easy visualization and interpretation of the results. For all of the plots, the FI has been plotted on the X-axis, the efficiency on the Y-axis and the cost/budget on the Z-axis.

Figure 6 shows the Pareto plot points for the cases when only high accuracy sensors were considered.

Figure 6 Pareto plot I

Figure 7, on the other hand, consists of the Pareto points obtained by considering all three types, i.e., low, medium and high accuracy sensors. From the two plots, it seems that 
higher levels of FI and efficiency are achievable when all three types of sensors are used in conjunction as expected.

Figure 7 Pareto plot II

Figure 7 Pareto plot II

Figure 8 Pareto plot III 
In Figure 8, the Pareto points from figures 6 and 7 have been overlapped, i.e., the Pareto points obtained by considering only high accuracy sensors have been plotted along with those obtained by considering all three types of sensors in the same graph. These plots are very useful to decision makers. For example, a decision maker wishes to achieve a high level of thermal efficiency at a low budget of around \$1 million will choose the selected points in the figure.

Figure 9 Pareto plot IV

To ensure that this is indeed true, we could rotate Figure 8 to obtain a two-dimensional view in Figure 9 where it can be seen that the selected point has a high thermal efficiency $(>42 \%)$. However, viewing the plot from another direction (figure 10)shows that 
the FI value is very low (close to 20 ). So, even though a budget of $\$ 1$ million would be sufficient to achieve a substantially high value of efficiency, it is unfortunately not enough to warrant a desirable value of FI. This suggests that the budget needs to be increased.

In addition to analyzing single points, it is also possible to analyze clusters of points at a time. This helps in eliminating certain solutions which do not show any clear advantage. For example, in Figure 11, there are two clusters of points, both containing points with budgets ranging from around $\$ 4$ million to $\$ 10$ million.

Figure 11 Pareto plot VI 
To analyze the performance of these two clusters in terms of efficiency, the 3-D plot in figure 11 is rotated to appear as shown in figure 12. It can be seen that for both the clusters, the efficiency is high (around 44\%)

However, when performance is compared in terms of FI, there is a significant difference between these two clusters. To visualize this, the plot is rotated once again as shown in figure 13. Now, we can clearly see that Cluster 1 on the left has low Fisher Information values ranging from 80-100 whereas cluster 2 on the right has a higher value of FI ranging from 130 to 160 . Thus, for the same budget, Cluster 2 points give us a higher value of FI and an equally good value of efficiency. Hence, Cluster 1 points can be eliminated from further consideration. The presence of these two clusters can be attributed to the fact that we have a non-convex problem.

Figure 13 Pareto plot VIII

Figure 13 Pareto plot VIII

Finally, it might be worthwhile to select Pareto points that perform reasonably well with respect to both FI and efficiency at a moderate budget.

Assuming a moderate budget of $\$ 5.275$ million is available and a high level of efficiency as well as observability is obtained, two solutions have been selected in Figure 14. The optimal sensor network design corresponding to these two points are shown below in 
Tables IV and V. Point 1 is obtained by using high accuracy sensors only, while point 2 is obtained by using all the three types of sensors. Thus, the Pareto set plots can be used to determine the most desirable solutions with respect to. to all the 3 objectives.

Point 1 has an efficiency of $44.56 \%$ and a FI of 144.04. A total of 13 locations have been selected where high accuracy sensors have to be physically placed and virtual sensing is to be used for the remaining 11 locations.

Figure 14 Pareto plot IX

Table IV: Sensor location for Point 1 (using only high accuracy sensors)

Point 2 has an efficiency of $43.77 \%$ and an FI of 154.81. As per this sensor network design, 10 locations require physical sensors with low accuracy, 3 with medium accuracy, 8 with high accuracy and the remaining 3 locations will be monitored virtually.

Table V: Sensor location for Point 2 (using low, medium \& high accuracy sensors)

From the list of the 24 intermediate variables in table VI, we can see that certain locations (star-marked) have been selected in both the solutions as requiring high accuracy physical sensors. This observation is rather intuitive since all of these variables are either inputs or outputs to the gasifier. Thus they have a huge impact on the overall plant performance because they are associated with the largest and most important equipment and/or process of the IGCC plant. 


\section{Table VI - list of intermediate variables}

Analyzing the Pareto surfaces has shed light upon some important observations. First, it is cheaper to maximize efficiency than it is to maximize FI. This is expected because fewer sensors need to be installed to reduce measurement uncertainties for process variables that have an effect on the thermal efficiency of the plant. Secondly, these graphs shows an increasing trend in the budget may not result in a proportional increase in FI or efficiency. It is evident from Figure 9 that beyond a budget of $\$ 5.27$ million, the values of efficiency become almost constant at about $44 \%$. A similar stagnation can be seen in the case of FI (around 160) at a budget of $\$ 7.38$ million. These values can vary subject to changes in the market price or measurement accuracy of the sensors used. However, the conclusion that increasing the budget beyond a certain point is futile since an equivalent improvement in the desired objective functions will not be achieved will always stand true.

\section{SUMMARY \& FUTURE WORK}

In an IGCC plant operation, maximizing observability, maximizing plant thermal efficiency, and minimizing the cost of sensor deployment are all desirable goals for 
stakeholders. In this research, all of these three objectives were considered jointly. This, along with the presence of measurement uncertainties and integer decision variables, led to the formulation of a nonlinear, stochastic, mixed integer multi-objective optimization problem. The aim was to deploy a combination of virtual sensors(based on simulation models built in ASPEN Plus) and real sensors in the IGCC plant to meet the above mentioned objectives.

This computationally intensive problem was solved using a novel algorithmic framework based on two-tier constraint method and the BONUS algorithm, which is an extremely efficient algorithm for nonlinear stochastic problems. By eliminating the need to run the sampling loop and simulation model for subsequent iterations, BONUS reduced the computational time of the problem (based on 800 samples) by more than $99 \%$.

To solve the multi-objective optimization problem, a 2-tier constraint method was used to generate pay-off tables for various cost bins. Pareto surfaces were generated to depict multiple feasible solutions that provided trade-offs between all the three objectives. Appropriate weightage for each of the three objectives can be decided based upon financial, environmental or other considerations. As the budget increases both thermal efficiency as well as Fisher Information increase. Yet it has been observed that it is cheaper to increase the former as compared to the latter. Moreover, even if both objective functions need to be maximized simultaneously, the budget does not need to be increased indefinitely. This is because beyond a particular amount, the growth of either of the objective functions essentially stagnates.

The key contributions of our paper are twofold. First, by solving a multi-objective problem, we have made it possible to bring into perspective several objective functions that are essentially different and yet related for improving the operating ability of an real world IGCC plant. Secondly, a new and computationally efficient algorithmic framework based on 2-tier constraint method and the BONUS algorithm was applied to solve this massive sensor network design problem. The significant reduction in computational time brings about a lot of flexibility in the problem by making it possible to process multiple scenarios and designs in a short amount of time.

Due to these significant contributions, this research has laid down the groundwork for further exploration of the sensor network design problem in the future. One particular avenue of research could be comparing the performance of the stochastic programming approach used in this research with that of dynamic simulation methods used by Paul et al. (28). The computational time using the former approach based on BONUS is expected to be much lower, in minutes while the latter approach takes hours to find solutions for a single case.

The computational efficiency of BONUS makes it suitable for real time optimization of dynamic sensor networks such as used in object tracking. (41) Also, the multi-objective optimization problem could be broadened to consider other objective functions such as maximizing the $\mathrm{CO}_{2}$ efficiency in the AGR unit of the IGCC plant. 
Finally, the application of these methodology could be extended to other problems which deal with other black box systems, i.e., a system whose outputs can be derived from its inputs via mathematical computation or programming without considering the internal workings of the system. For example, other chemical plants such as bioreactors, gas processing facilities etc, could use this technology. Black box models exist in a myriad of areas at present such as psychology, cryptography etc. So, an ambitious objective would be to apply a modified version of the solution methodology developed in this research to black box models in areas that are not closely related to chemical engineering.

\section{REFERENCES}

[1] Sahin K, Diwekar UM. "Better optimization for nonlinear uncertain systems (BONUS): a new algorithm for stochastic programming using reweighting through kernel density estimation." Ann Oper Res 2004; 132:47-68.

[2] Diwekar U., David A., "BONUS Algorithm for Large Scale Stochastic Non-linear Stochastic Algorithm Problems." Springer, 2015.

[3] He F, Li Z, Liu P, Maa L, Pistikopoulos EN. "Operation window and part-load performance study of a syngas fired gas turbine.” Applied Energy 2012;89:133-41.

[4] Park SK, Ahn J, Kim TS. "Performance evaluation of integrated gasification solid oxide fuel cell/gas turbine systems including carbon dioxide capture." Applied Energy 2011; 88:2976-87.

[5] Pérez-Fortes M, Bojarski AD, Puigjaner L. "Advanced simulation environment for clean power production in IGCC plants.” Comput Chem Eng 2011; 35:1501-20.

[6] Meo, M., Zumpano G. "On the optimal sensor placement techniques for a bridge structure," Engineering Structures 2005; 27:1488-97.

[7] Liu, W., W.-C. Gao, Y. Sun, M.-J. Xu, "Optimal sensor placement for spatial lattice structure based on genetic algorithms," Journal of Sound and Vibration 2008; 37:175-189.

[8] Das, S., A. Chattopadhyay, X. Zhou, "Acoustic Based Structural Health Monitoring for Composites Using Optimal Sensor Placement: Analysis and Experiments," Journal of Reinforced Plastics and Composites 2009; 28:83-97. 
[9] Berry J., Fleischer L., Hart W., Philips C., J. Watson, "Sensor placement in muncipal water networks," J. Water Resour. Plan. Manage. 2005; 131(1):237-243.

[10] Shastri Y., Diwekar U., "Sensor placement in water networks: A stochastic programming approach", Journal of Water Resources, Planning and Management, 2006; 132:192-203.

[11] Rico-Ramirez V., Frausto-Hernandez S., U. Diwekar, and S. Hernandez-Castro, "Water networks security: A two-stage mixed-integer stochastic program for sensor placement," Computers and chemical Engineering 2007; 31:565-573.

[12] Ban, X., L. Chu, R. Herring, J. D. Margulici, "Optimal Sensor Placement for Both Traffic Control and Traveler Information Applications," Transportation Research Board 88th Annual Meeting, Paper \#09-3315, 2009.

[13] Zhang, T., Q. Chen, C.-H. Lin, "Optimal sensor placement for airborne contaminant detection in an aircraft cabin," HVAC \& R Research, 2007.

[14] Wu, Q.; A. J. Chung; G.-Z. Yang, "Optimal sensor placement for predictive cardiac motion modeling," MICCAI: International Conference on Medical Image Computing and ComputerAssisted Intervention, 9(Pt 2), pp. 512-519, 2006.

[15] Ali Y, Narasimhan S. "Sensor network design for maximizing reliability of linear processes." AIChE J 1993;39(5):820-8.

[16] Alonso AA, Kevrekidis IG, Banga JR, Frouzakis CE. "Optimal sensor location and reduced order observer design for distributed process systems." Comput Chem Eng 2004; 28:27-35.

[17] Singh A., Hahn J., "Sensor location for stable non-linear systems: placing multiple sensors".

Ind. Eng. Chem. Res., 2006; 45 (10): 3615-23

[18] D’Souza K., Epureanu BI, "Sensor Detection for damage detection in non-linear systems using system augmentations", 2008; AIAA Journal, 2008; 46 (10): 2434-42

[19] Colantuoni G, Padmanabhan L. "Optimal sensor location for tubular-flow reactor systems." Chem Eng Sci 1977;32(9):1035-49.

[20] Harris TJ, Macgregor JF, Wright JD. "Optimal sensor location with an application to a packed bed tubular reactor." AIChE J 1980;26(6):910-6.

[21] Jorgensen SB, Goldschmidt L, Clement K. "A sensor location procedure for chemical processes." Comput Chem Eng 1984;8(3-4):195-204.

[22] Kumar S, Seinfeld SH. "Optimal location of measurements in tubular reactors." Chem Eng Sci 1978;33(11):1507-16.

[23] Omatu S, Koide S, Soeda T. "Optimal sensor location for a linear distributed parameter system.” IEEE Trans Autom Control 1978;23(4):665-73.

[24] Romagnoli J, Alvarez J, Stephanopolus G. "Variable measurement structures for process control." Int J Control 1981;33(2):269-89.

[25] Chang CT, Mah KN, Tsai CS. "A simple design strategy for fault monitoring systems." AICHE J 1993;39(7):1146-63.

[26] Kelly JD, Zyngier D. "A new and improved MILP formulation to optimize observability, redundancy and precision for sensor network problems." AIChE J 2008;54(5):1282-91.

[27] Madron F, Veverka V. "Optimal selection of measuring points in complex plants by linear models.” AIChE J 1992;38(2):227-36.

[28] Ragot J, Maquin D. "Fault measurement detection in an urban water supply network." J Process Cont 2006;16(9):887-902. 
[29] Bagajewicz MJ. "Design and retrofit of sensor networks in process plants." AIChE J 1997; 43:2300-6.

[30] Chmielewski DJ, Palmer T, Manousiouthakis V. "On the theory of optimal sensor placement.' AIChE J 2002;48(5):1001-12.

[31] Muske KR, Georgakis C. "Optimal measurement system design for chemical processes." AIChE J 2003;49(6):1488-94.

[32] Seenumani G, Dai D, Lopez-Negrete R, Kumar A, Dokucu M, Kumar R. "An OuterApproximation Based Algorithm for Solving Integer Non-linear Programming Problems for Optimal Sensor Placement." $51^{\text {st }}$ IEEE Conference on Decision and Control 2012;4455-61.

[33] Lee, A. Diwekar, U. 'Optimal sensor placement in integrated gasification combined cycle power systems”. Applied Energy 2012; 99:255-264.

[34] Paul P, Bhattacharyya D, Turton R, Zitney SE. "Sensor Network Design for Maximizing Process Efficiency: An Algorithm and its Application." AIChE J 2015;61(2):464-76.

[35] Iqbal M., Naeem M., Anpalagan A., Ahmed A., Azam M. "Wireless Sensor Network Optimization: Multi-Objective Paradigm". Sensors, 2015;7:17572-17620

[36] Stehlik M., Saleh A., Stetsko A., Matyas V. "Multi-objective Optimization of Intrusion Detection Systems for Wireless Sensor Networks." Advances in Artificial Life, ECAL 2013; 12:569-76

[37] Aral MM, Guan J, Maslia ML. "A Multi-Objective Optimization Algorithm for Sensor Placement in Water Distribution Systems." World Environmental and Water Resources Congress, 2008: 1-11.

[38] Tampa electric polk power station integrated gasification combined cycle report. Final technical report, <http://www.tampaelectric.com/data/files/PolkDOEFinalTechnicalReport.pdf>. August 2002.

[39] Holt, NAH., "Integrated Gasification Combined Cycle Power Plants." Encyclopedia of Physical Science and Technology, 2001; $3^{\text {rd }}$ edition.

[40] Kabadayi S., Pridgen A., Julien C. "Virtual Sensors: Abstracting data from physical sensors" Proceedings of the 2006 international symposium on world of wireless, mobile and multimedia networks, 2006; 587-592.

[41] GE, Integrated Sensing \& Controls for Coal Gasification - Development of Model-Based Controls for GE’s Gasifier \& Syngas Cooler. 2008 Q1 (Jan-Mar) Report to NETL. April 2008

[42] Fisher RA. "On the mathematical foundation of theoretical statistics." Philos Trans Roy Soc Lond, 1922; 222:309-68.

[43] Frieden BR, Gatenby RA. "Exploratory data analysis using fisher information." Springer Science \& Business Media, 2010.

[44] Masi M. "Generalized information-entropy measures and Fisher information." NASA Astrophys Data Syst 2008; 1-16.

[45] Diwekar, U., and Kalagnanam J. "Robust design using an efficient sampling technique." Computers \& chemical engineering 1996; 20:389-94.

[46] Kalagnanam J. and U. Diwekar, "An efficient sampling technique for off-line quality control”, Technometrics 1997; 39:308-319. 


\section{A Multi-Objective Optimization Approach to Optimal Sensor Location Problem in IGCC Power Plants: List of Figures}

- Figure 1 - A simplified process flow diagram of the IGCC process plant. (Source: (29))

- Figure 2 - Potential sensor locations in the IGCC power plant (Source: (29

- Figure 3 - Pictorial representation of the numerical optimization framework (Source: Diwekar and David [5])

- Figure 4 - Pictorial representation of the stochastic programming framework (Source:[5])

- Figure 5 - Pictorial representation of reweighting scheme(Source:[5])

- Figure 6: Pareto plot I: considering all high accuracy sensors.

- Figure 7: Pareto plot II: considering high, medium, and low accuracy sensors.

- Figure 8: Pareto plot III: overlapping figures $7 \& 8$

- Figure 9: Pareto plot IV, two dimensional view of figure 8, view 1

- Figure 10: Pareto plot $V$, two dimensional view of figure 8, view 2

- Figure 11: Pareto plot VI, clusters of point medium to high cost

- Figure 12: Pareto plot VII, two dimensional view figure 11, view 1

- Figure 13: Pareto plot VIII, two dimensional view figure 11, view 2

- Figure 14: Pareto plot IX, moderate budget Pareto points 


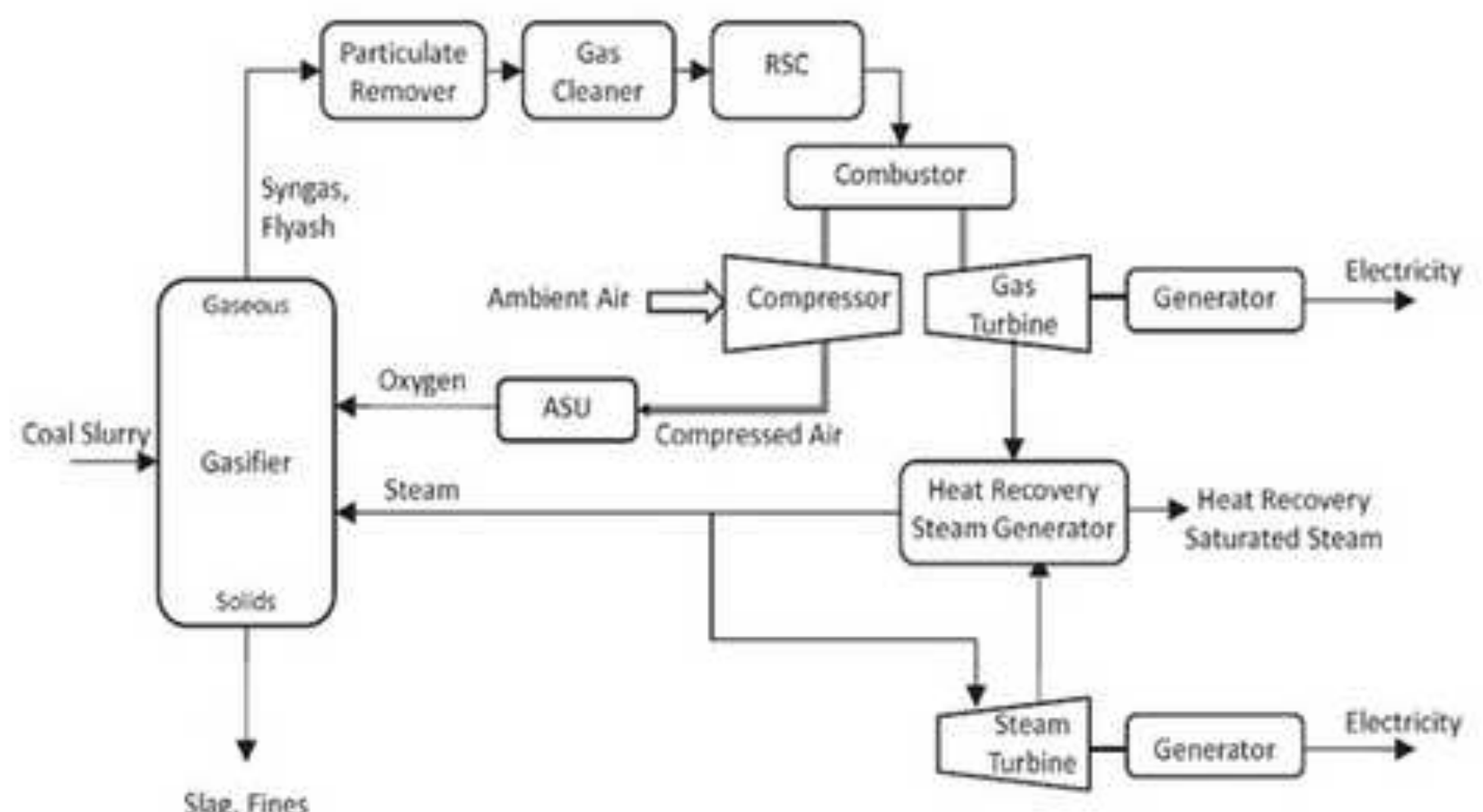

Slag, Fines 


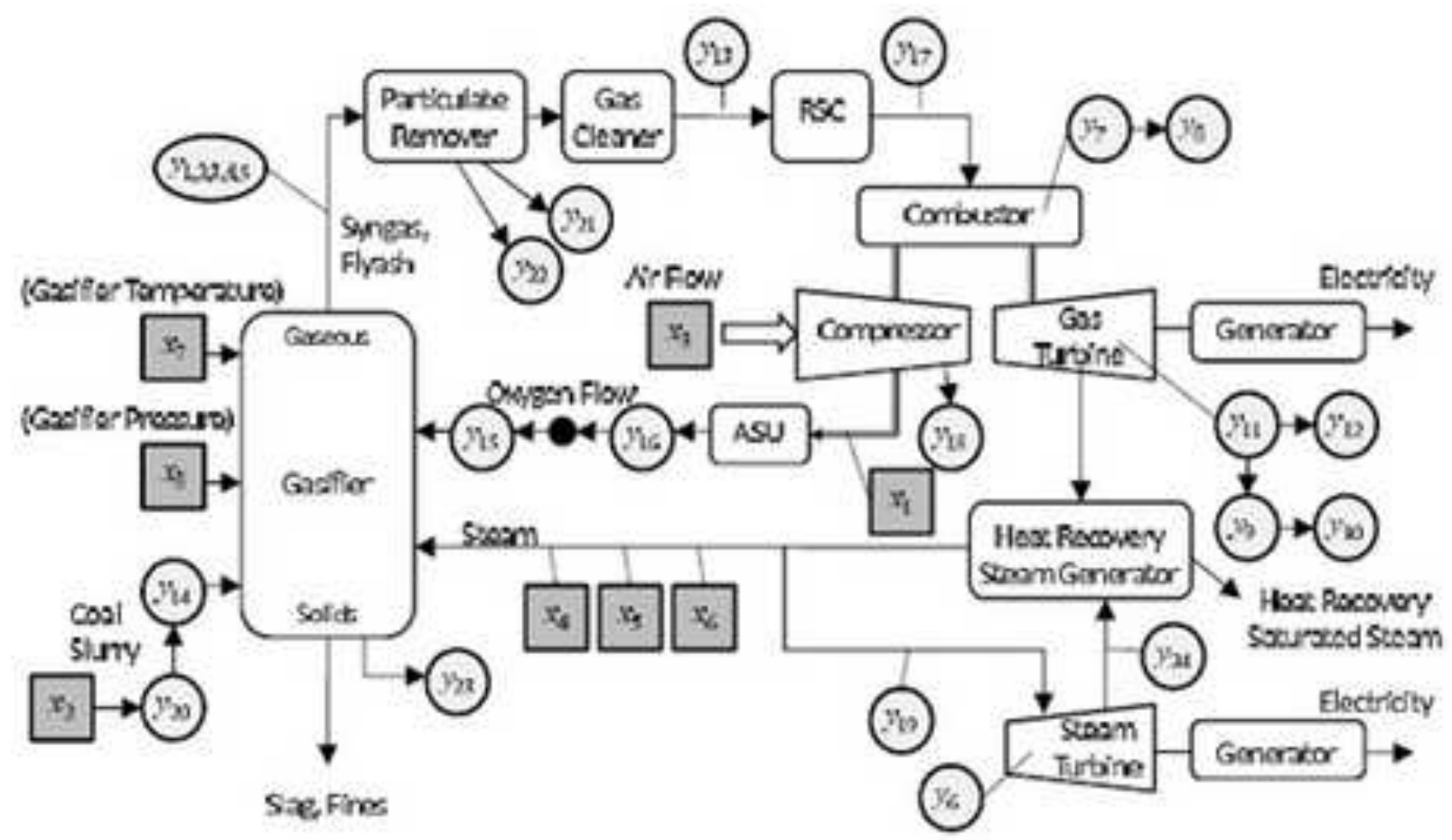




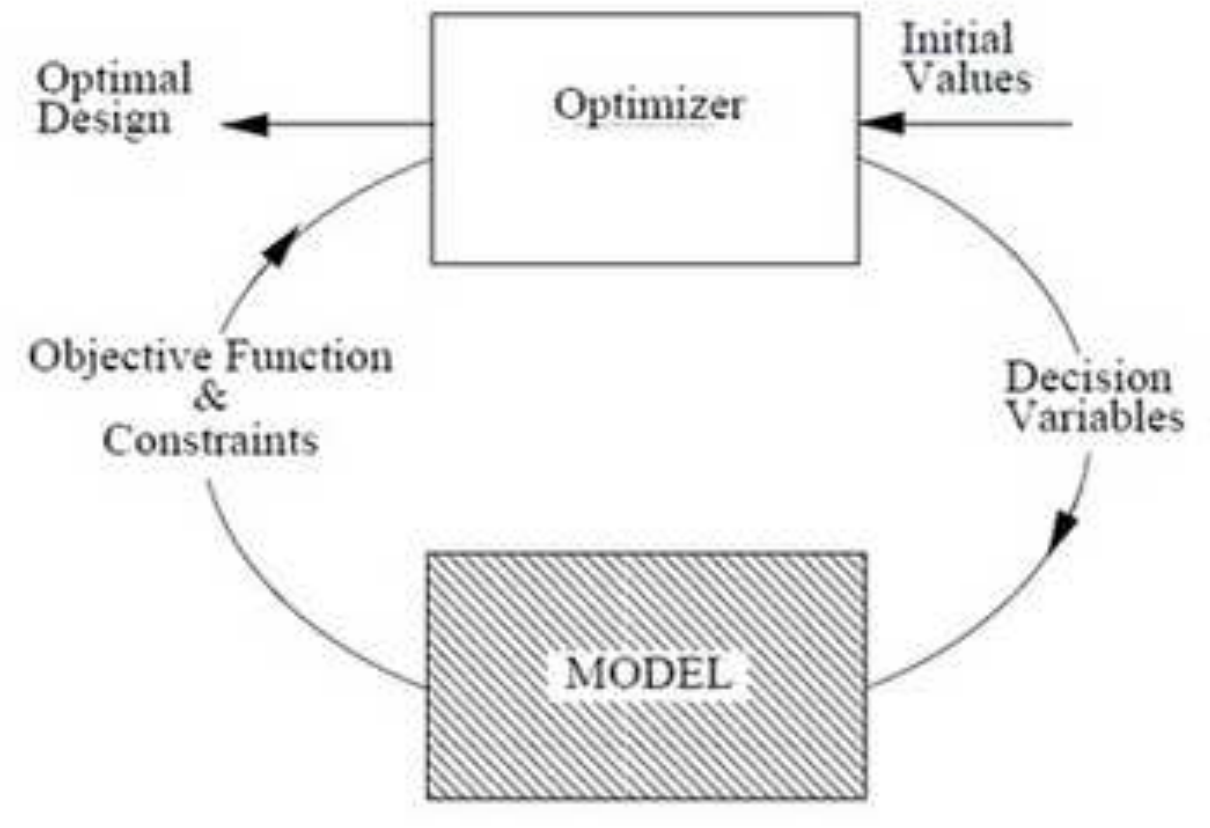

objective Function

Constraints

Decision

ariables 


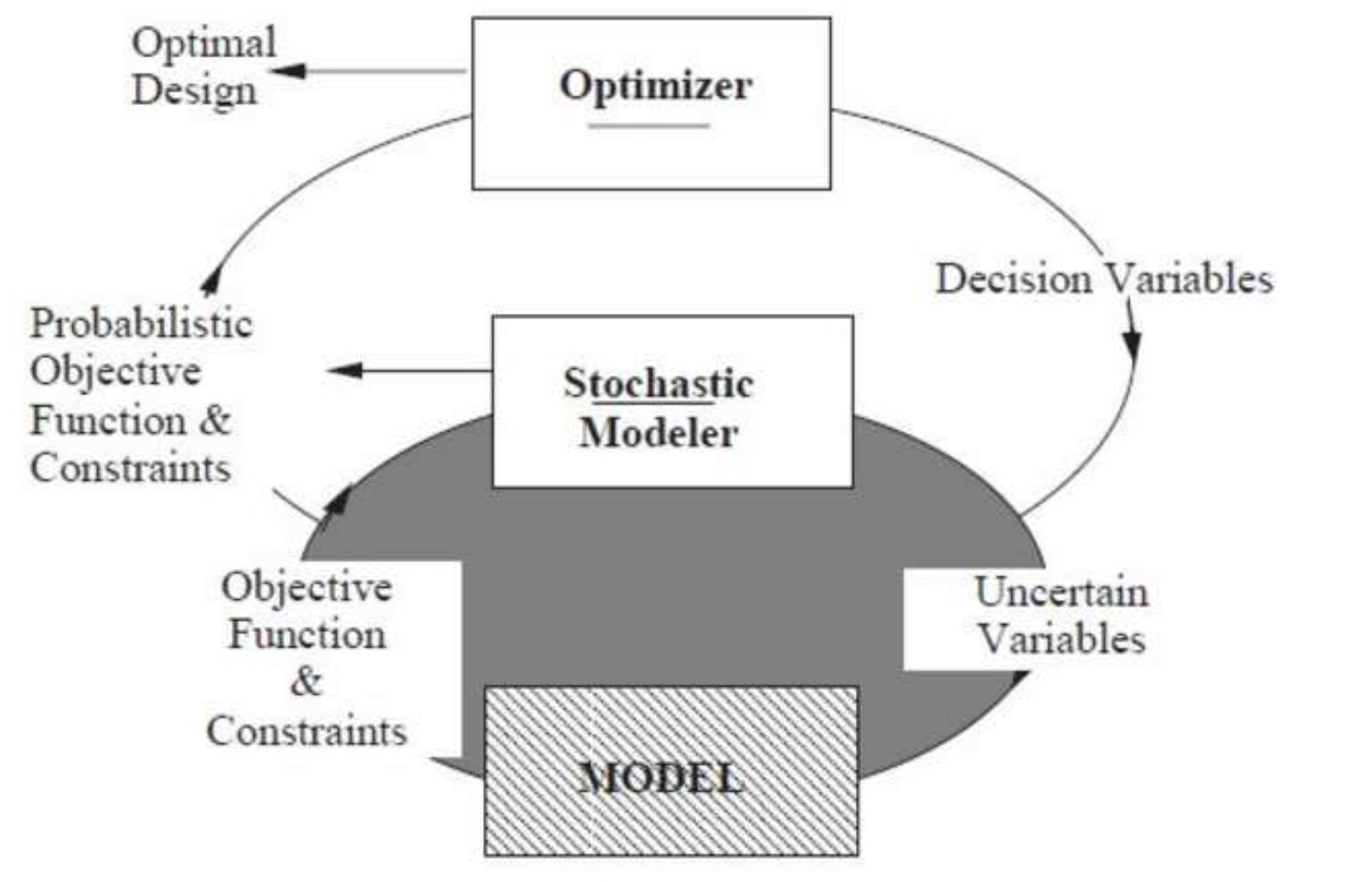

Probabilistic Objective Function \& Constraints

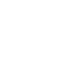
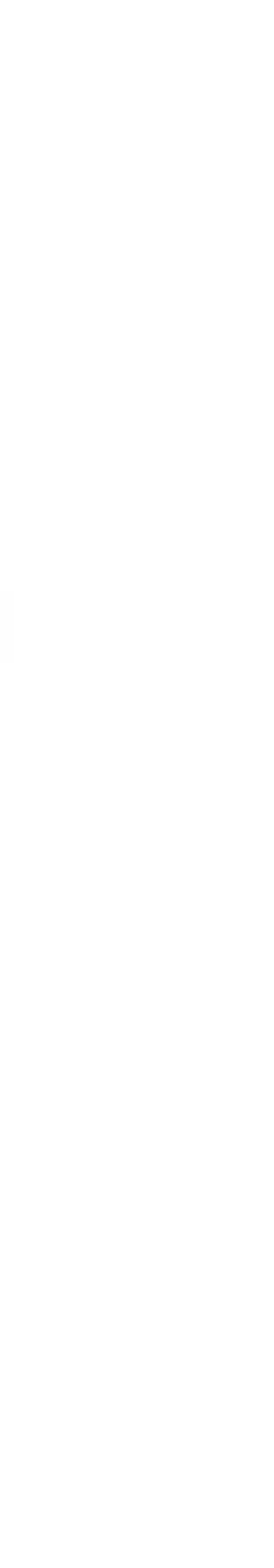

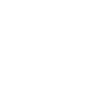
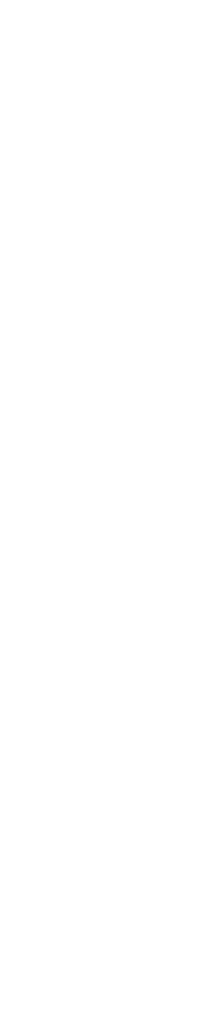


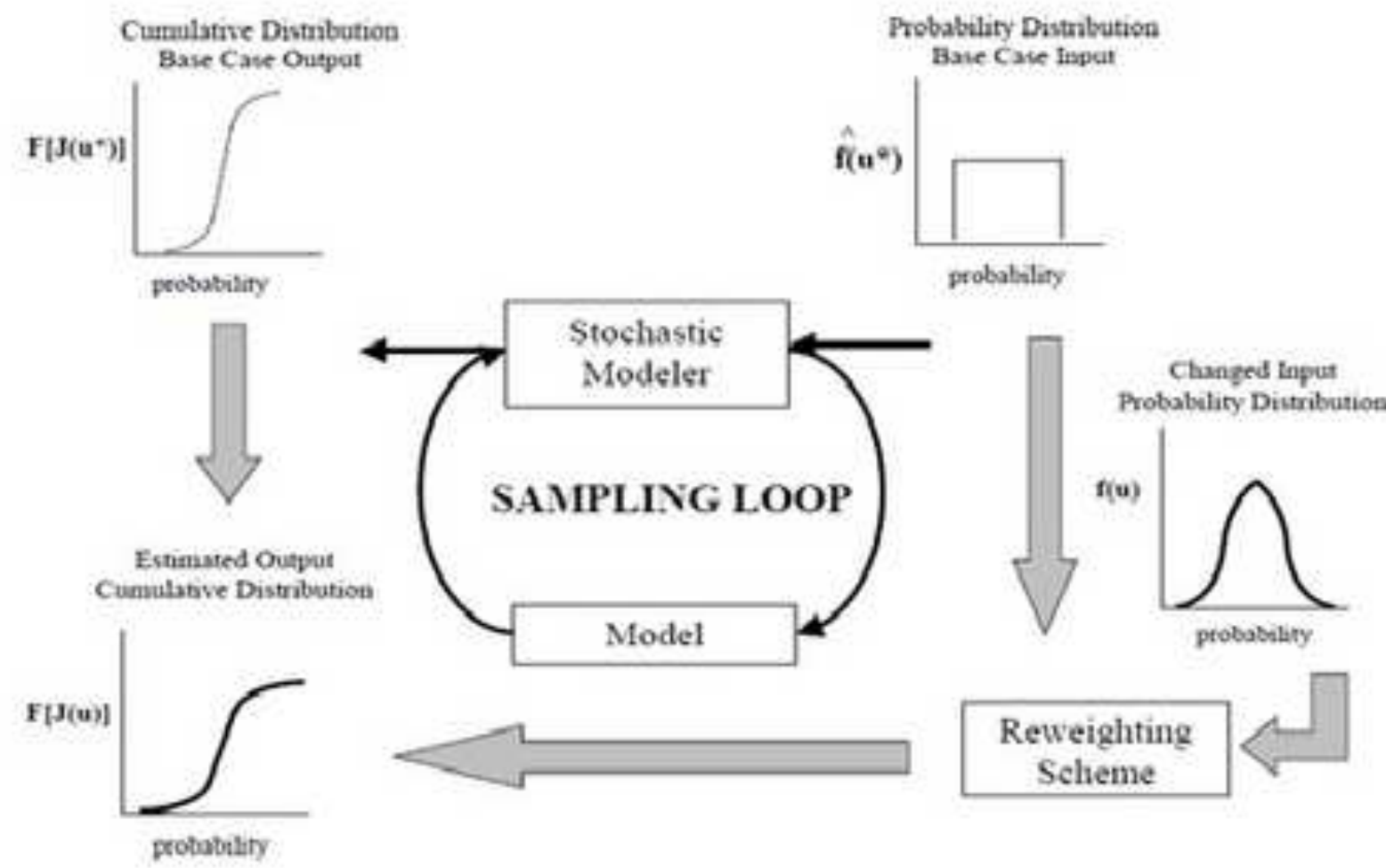




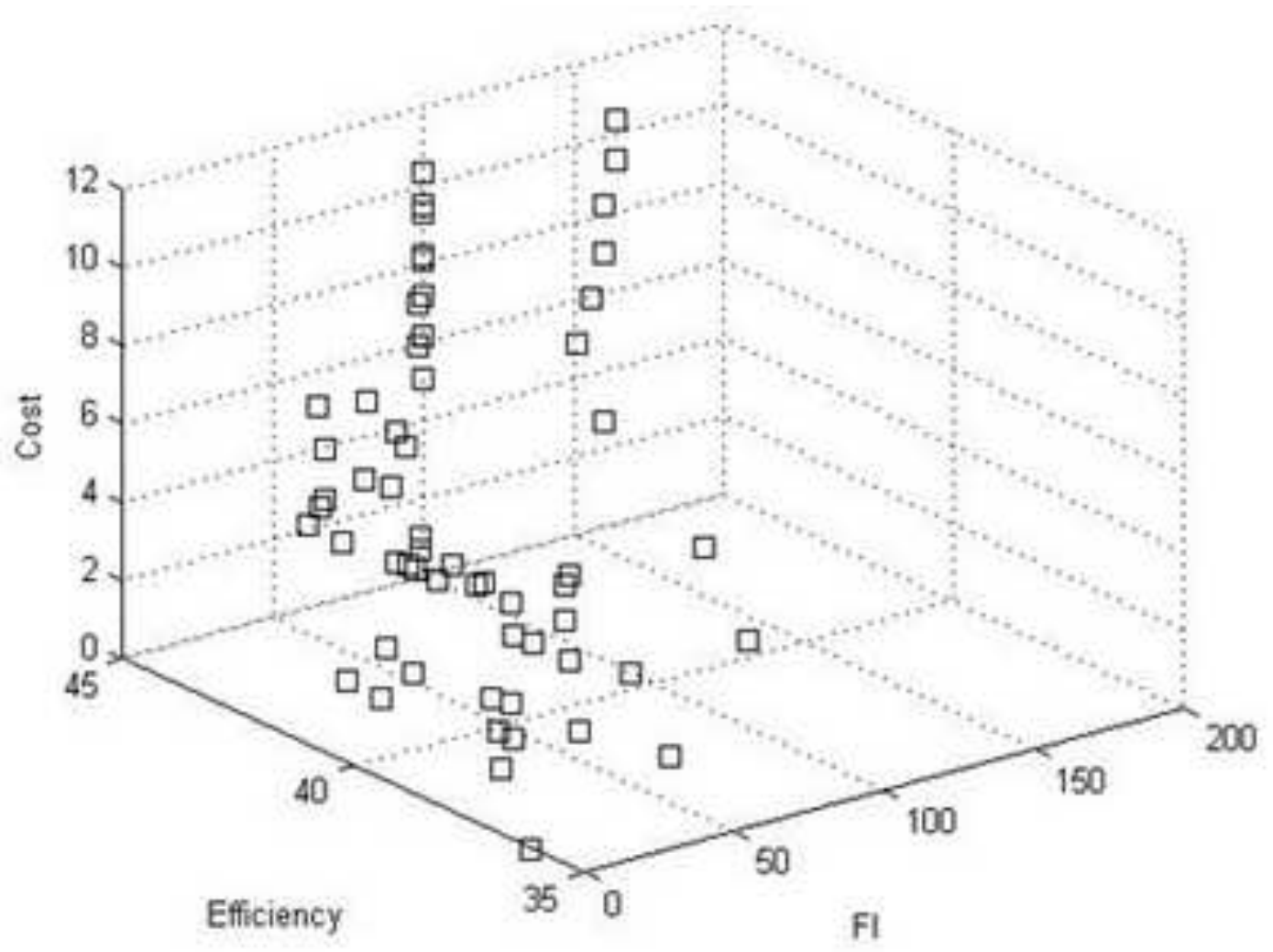




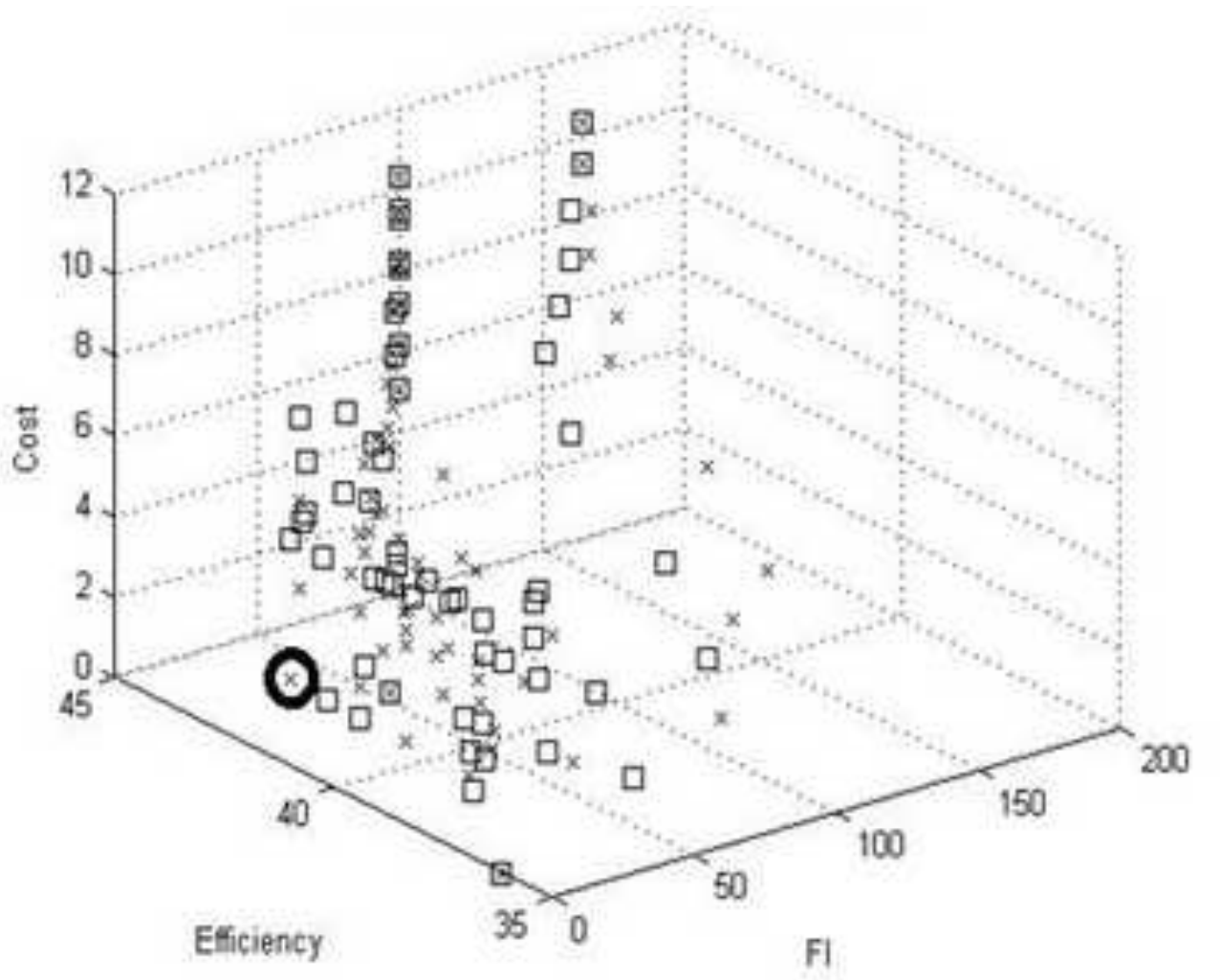




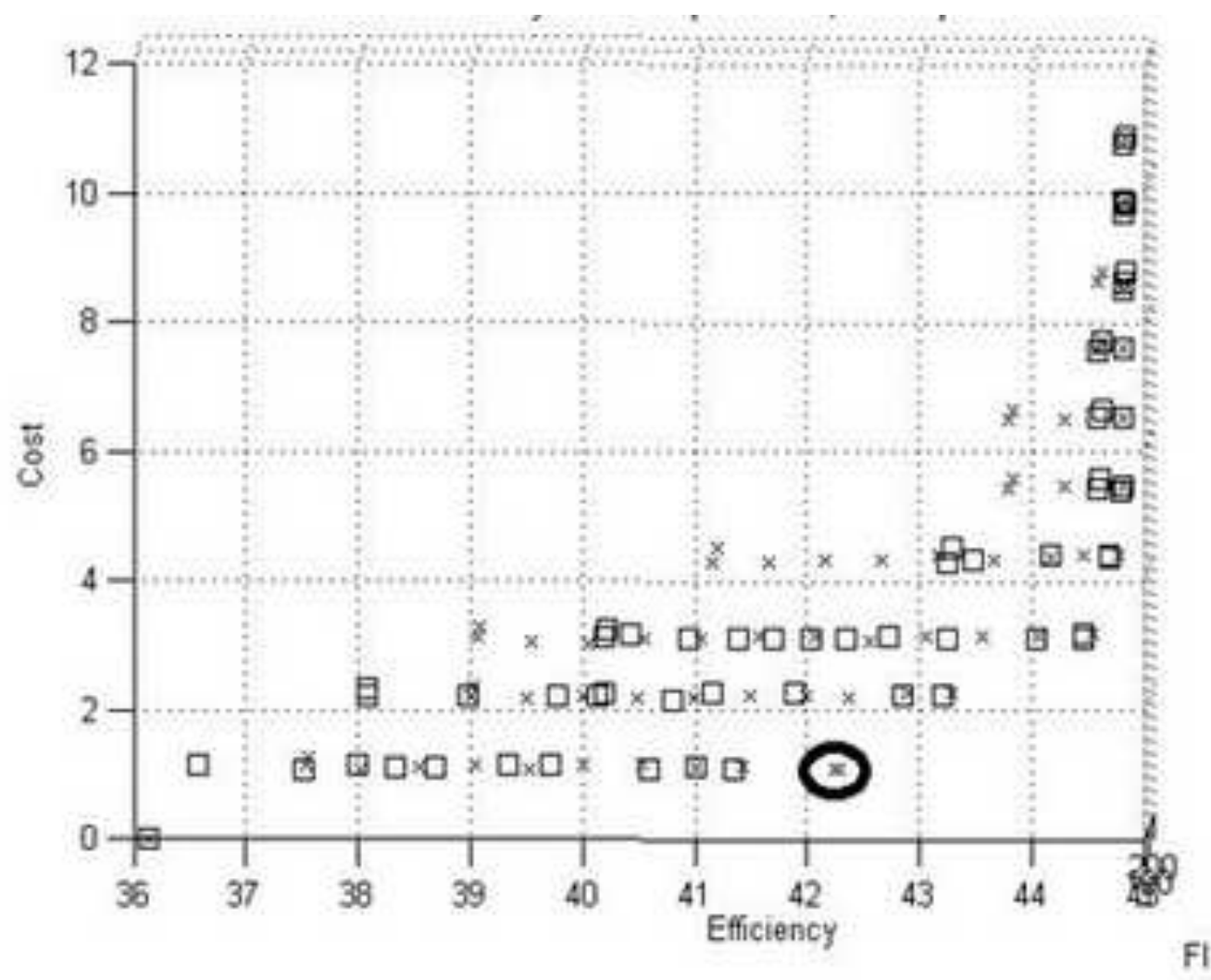




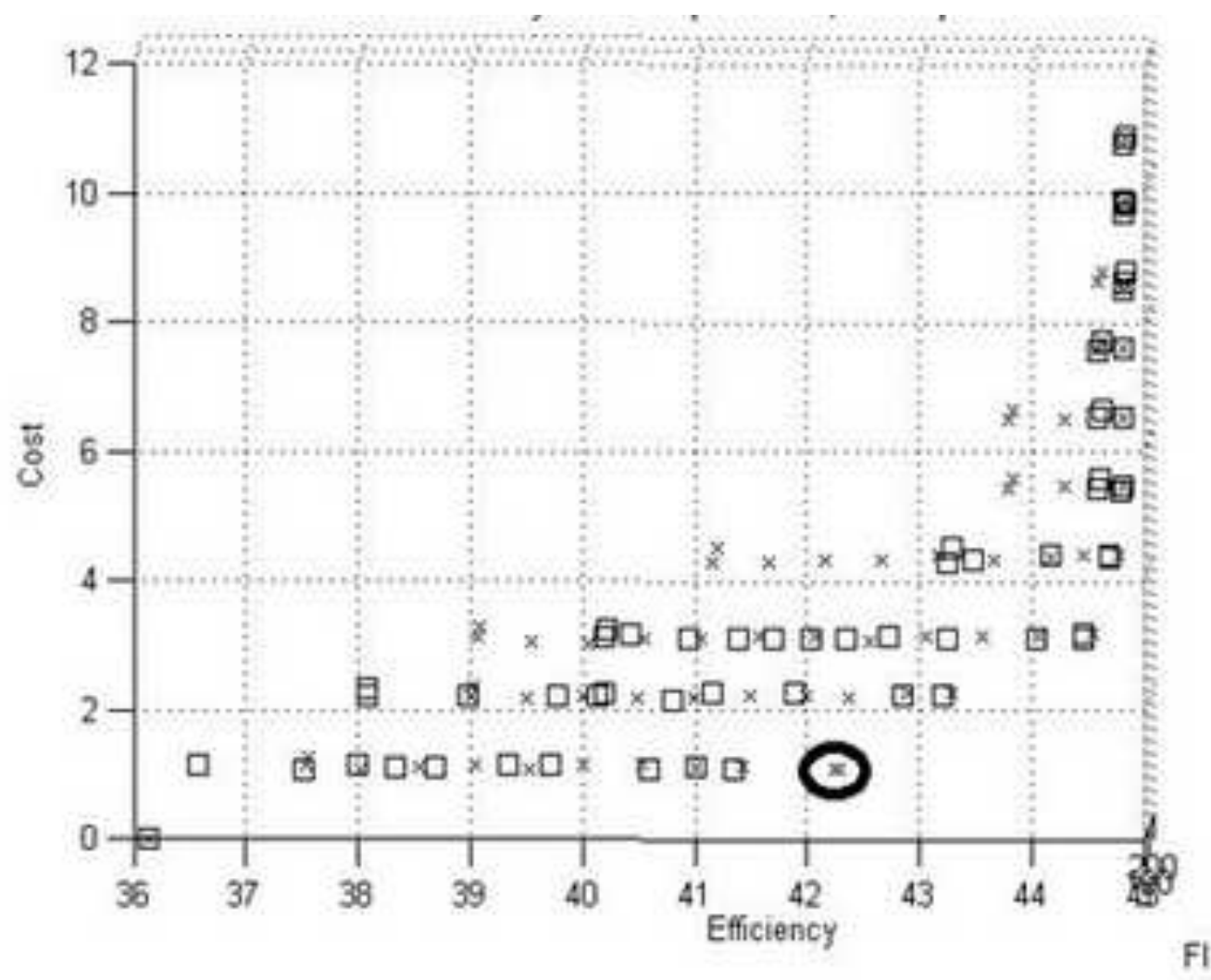




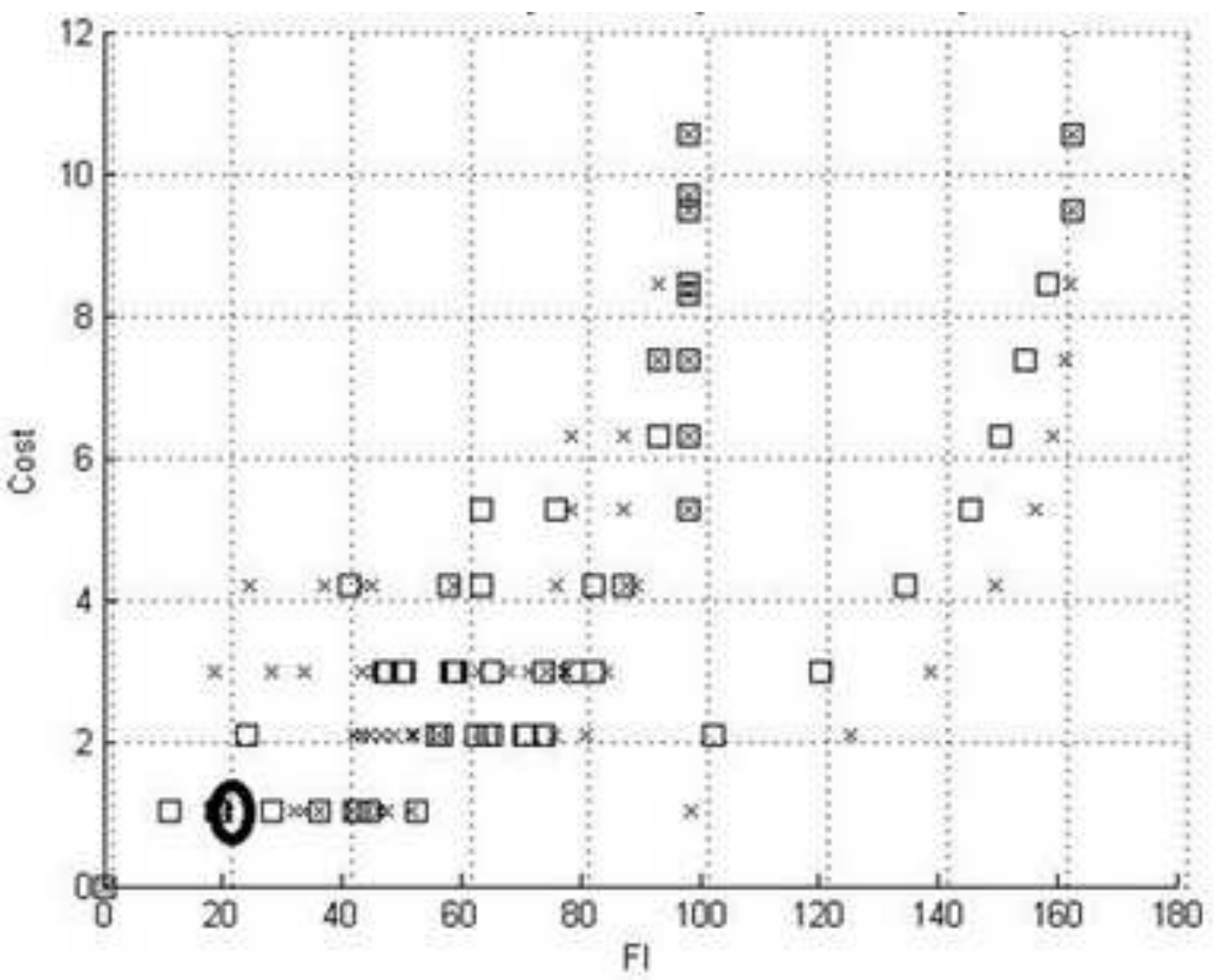




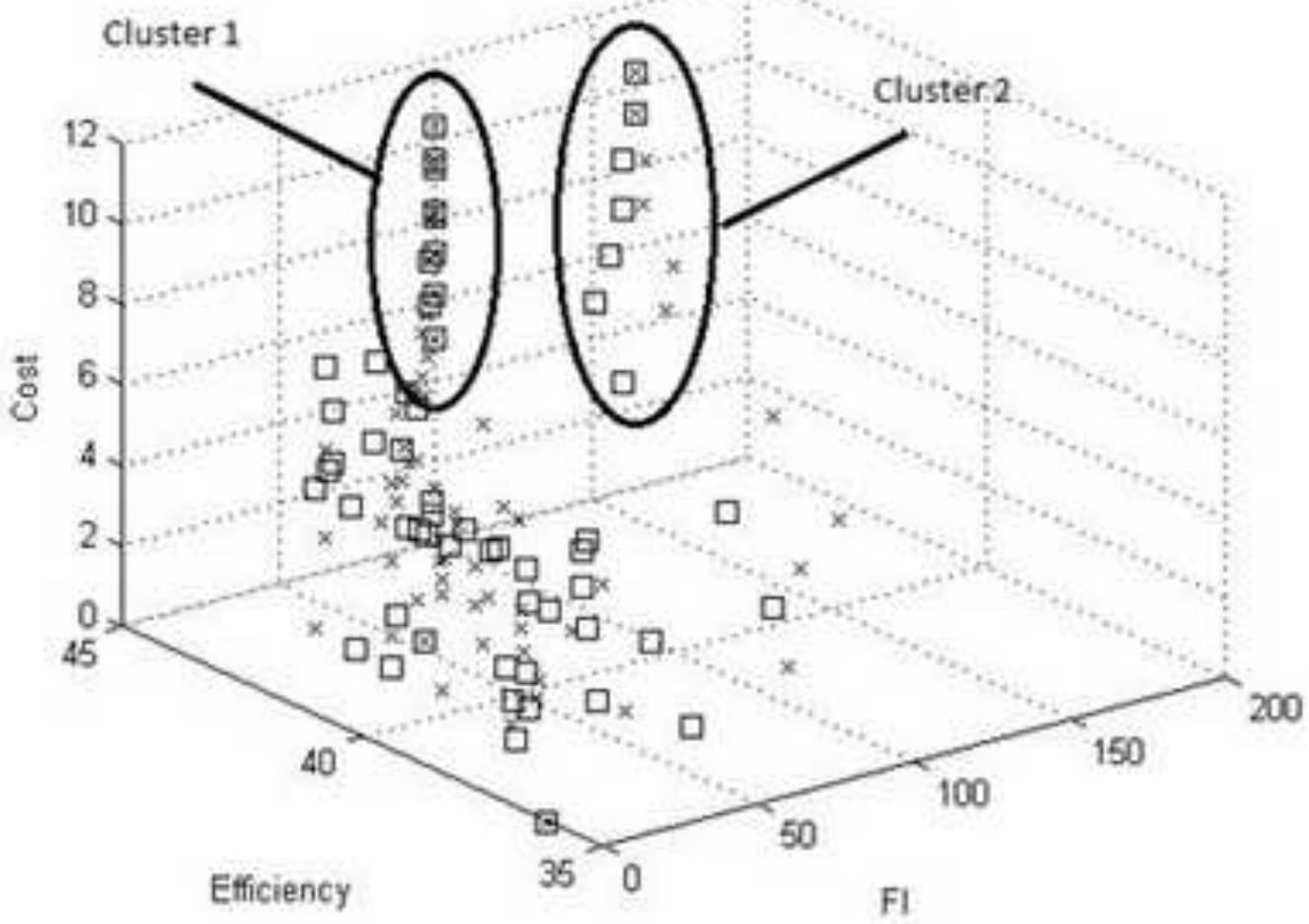




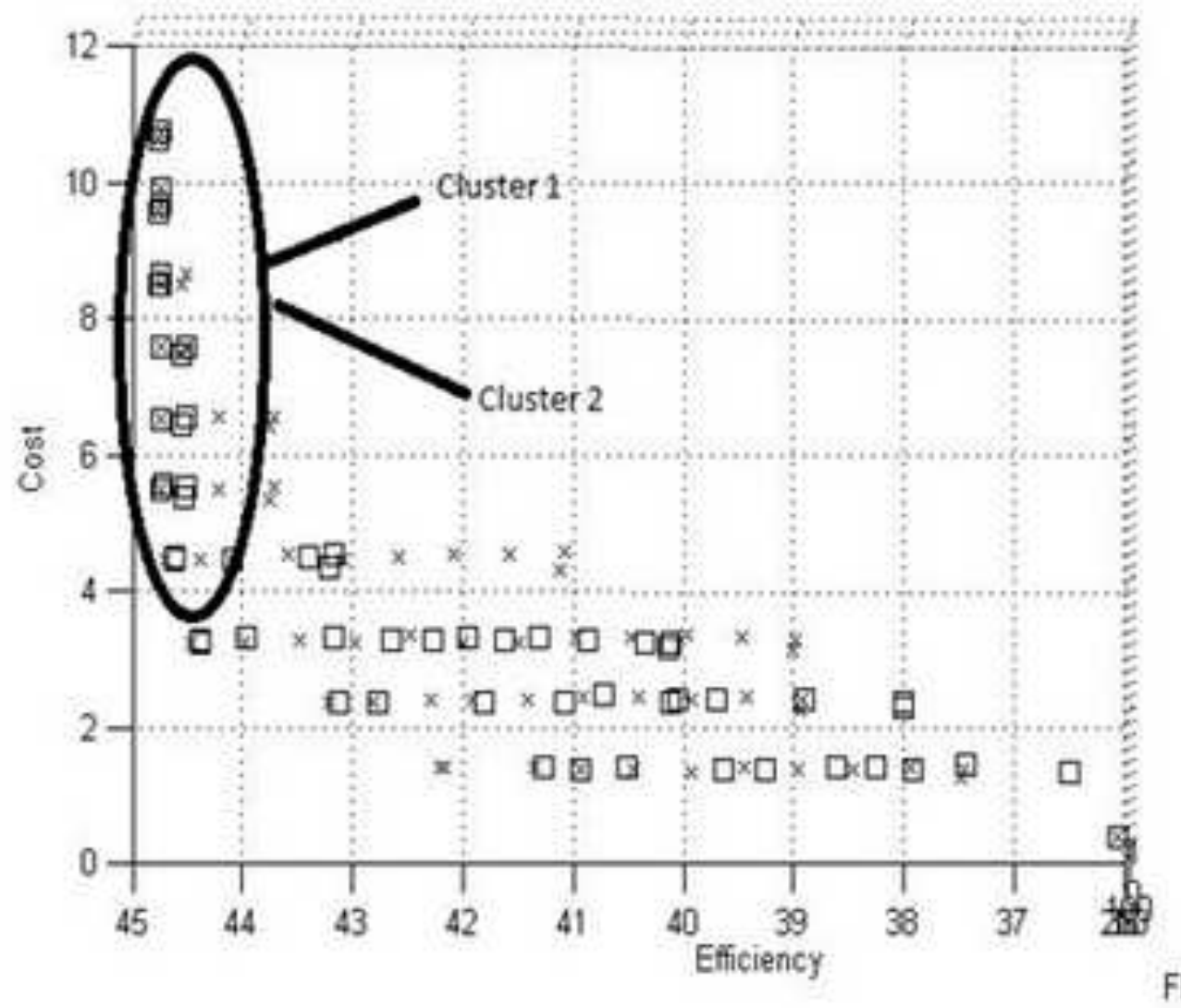




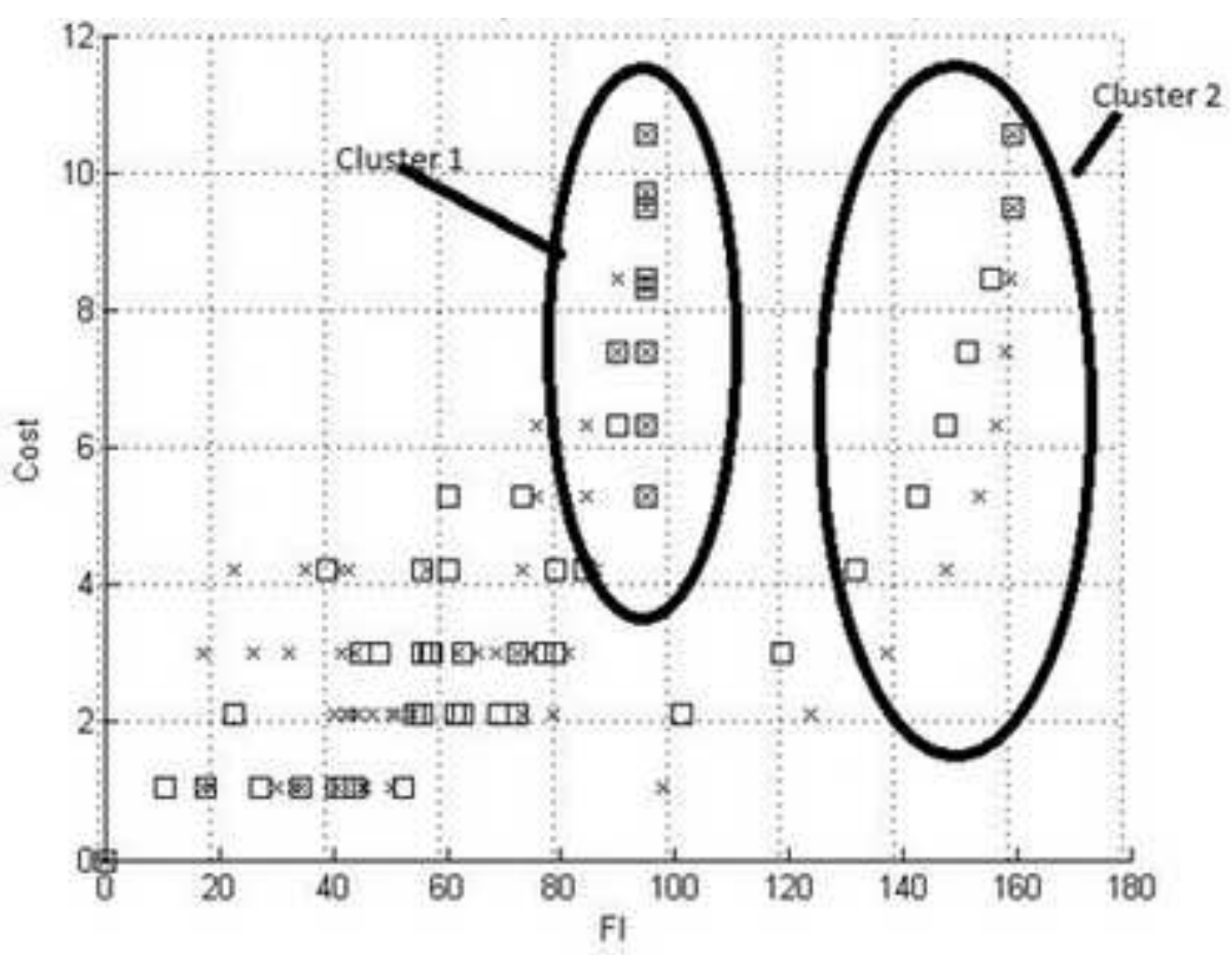




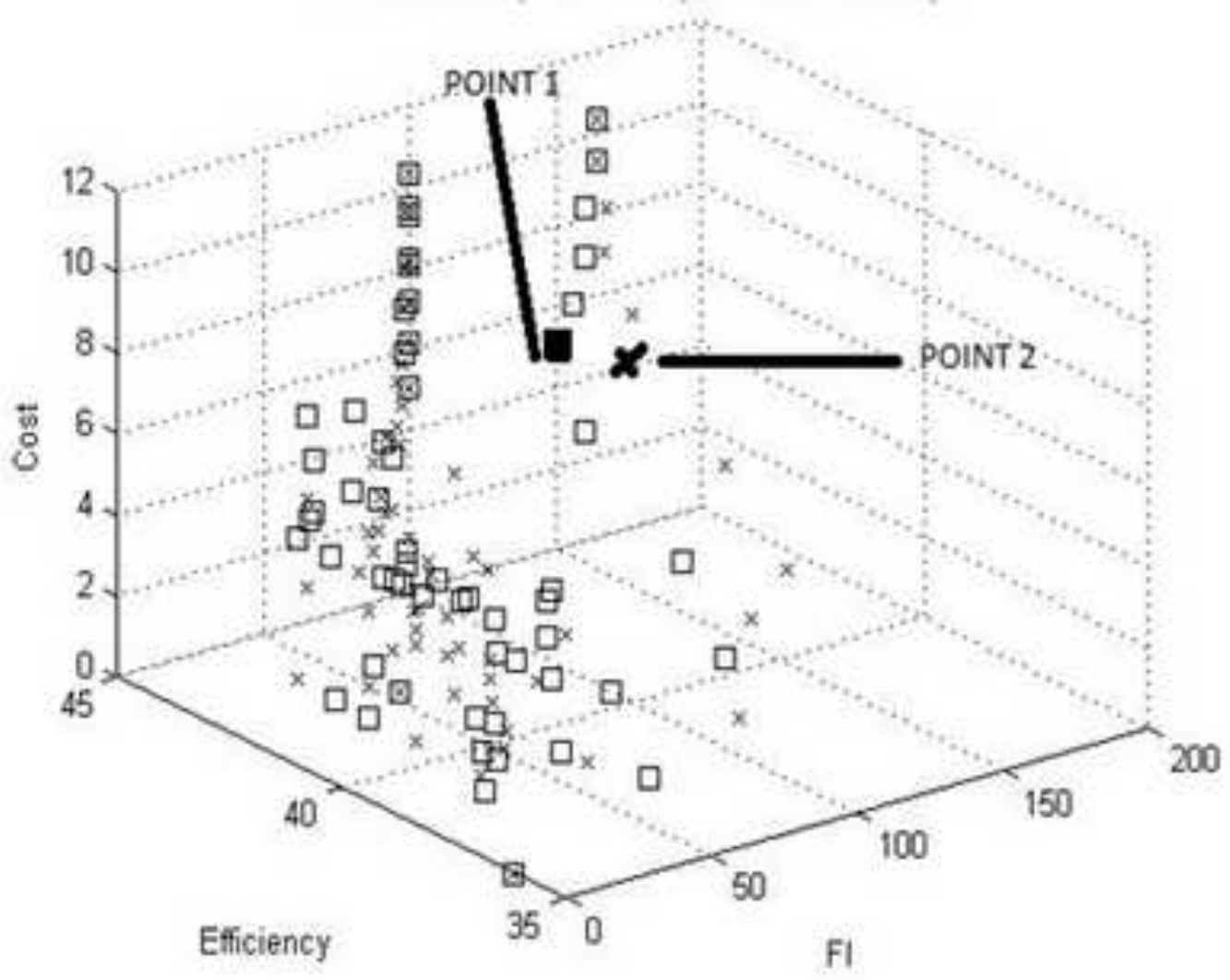




\begin{tabular}{|ll|}
\hline $\mathbf{Y}_{\mathbf{i}}$ & Description \\
\hline $\mathbf{1}$ & Gasifier syngas flow rate \\
\hline $\mathbf{2}$ & Syngas CO flow rate \\
\hline $\mathbf{3}$ & Syngas $\mathrm{CO}_{2}$ flow rate \\
\hline $\mathbf{4}$ & Syngas temperature \\
\hline $\mathbf{5}$ & Syngas pressure \\
\hline $\mathbf{6}$ & Low pressure steam turbine temperature \\
\hline $\mathbf{7}$ & Gas turbine combustor burn temperature \\
\hline $\mathbf{8}$ & Gas turbine combustor exit temperature \\
\hline $\mathbf{9}$ & Gas turbine high pressure exhaust stream temperature \\
\hline $\mathbf{1 0}$ & Gas turbine low pressure exhaust stream temperature \\
\hline $\mathbf{1 1}$ & Gas turbine expander output temperature \\
\hline $\mathbf{1 2}$ & Flue gas flow rate exiting gas turbine expander \\
\hline $\mathbf{1 3}$ & Syngas flow rate after solids removal \\
\hline $\mathbf{1 4}$ & Coal slurry flow rate entering gasifier \\
\hline $\mathbf{1 5}$ & Oxygen flow rate into gasifier \\
\hline $\mathbf{1 6}$ & Oxygen flow rate exiting ASU \\
\hline $\mathbf{1 7}$ & Acid gas flow rate \\
\hline $\mathbf{1 8}$ & Gas turbine compressor leakage flow rate \\
\hline $\mathbf{1 9}$ & Flow rate into high pressure steam turbine \\
\hline $\mathbf{2 0}$ & Coal slurry feed flow rate \\
\hline $\mathbf{2 1}$ & Slag extracted from syngas \\
\hline $\mathbf{2 2}$ & Fines extracted from syngas \\
\hline $\mathbf{2 3}$ & Gasifier heat output \\
\hline $\mathbf{2 4}$ & Recvcled HRSG steam heat output \\
\hline & \\
\hline
\end{tabular}


Sensor Locations

\begin{tabular}{|c|c|c|c|c|c|c|c|c|c|c|c|c|c|c|c|c|c|c|c|c|c|c|c|c|c|c|c|}
\hline & & \multicolumn{25}{|c|}{ Sensor Locations } & \multirow[b]{2}{*}{ Efficiency } \\
\hline & Cost & П & 1 & 2 & 3 & 4 & 5 & 6 & 7 & 8 & 9 & 10 & 11 & 12 & 13 & 14 & 15 & 16 & 17 & 18 & 19 & 20 & 21 & 22 & 23 & 24 & \\
\hline 1 & 0 & 0 & - & - & - & - & - & - & - & • & - & - & - & - & • & • & . & - & * & - & • & - & - & • & - & - & 0.3514 \\
\hline 3 & 2110000 & 124.5 & H & L & L & . & M & - & - & - & L & $L$ & L & - & $\mathbf{u}$ & M & $\mathbf{M}$ & $\mathbf{M}$ & $\mathbf{M}$ & - & - & M & - & - & - & 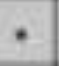 & 0.3898 \\
\hline 4 & 3000000 & 137.9 & H & $\mathbf{L}$ & L & $\mathbf{L}$ & $\boldsymbol{M}$ & - & L & $\cdot$ & L & $\mathbf{L}$ & $\mathbf{L}$ & . & $\mathbf{M}$ & $\mathbf{u}$ & $\mathbf{M}$ & $\mathbf{M}$ & M & L & - & $\boldsymbol{M}$ & - & - & - & 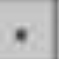 & 0.3904 \\
\hline 6 & 5275000 & 154.8 & H & M & L & $u$ & H & M & L & $\mathrm{L}$ & L & L & t & . & H & H & H & H & H & L & . & $\mathrm{H}$ & L & L & L & 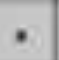 & 0.4377 \\
\hline 7 & 6330000 & 157.6 & H & M & M & M & H & H & $M$ & $\mathbf{M}$ & $L$ & L & M & - & H & H & H & H & H & L & * & H & L & L & L & * & 0.4377 \\
\hline 8 & 7385000 & 159.7 & H & H & M & H & H & M & M & M & L & $t$ & $\mathbf{M}$ & H & H & H & H & H & H & t & • & H & M & $\mathbf{u}$ & l & - & 0.4456 \\
\hline 9 & 8300000 & 160.5 & $\mathrm{H}$ & $\mathrm{H}$ & H & H & H & H & H & M & $\mathbf{M}$ & $\mathbf{M}$ & H & H & $\mathrm{H}$ & H & H & H & H & M & - & H & $M$ & $\mathbf{M}$ & L & * & 0.4456 \\
\hline
\end{tabular}

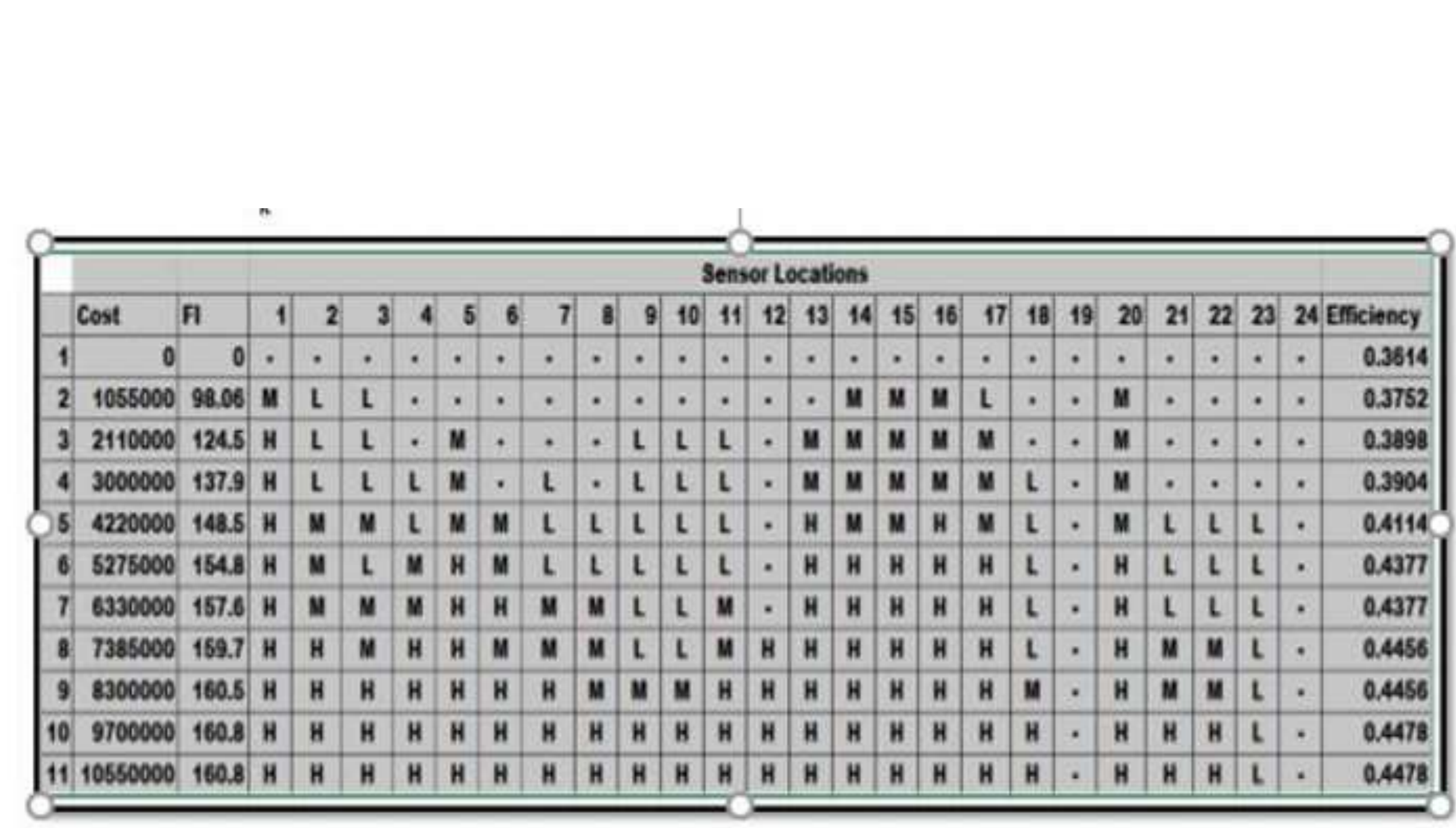




\begin{tabular}{|l|l|l|l|l|l|l|l|l|l|l|l|l|l|l|l|l|l|l|l|l|l|l|l|l|l|l|}
\hline \\
\hline
\end{tabular}

\footnotetext{
西

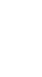

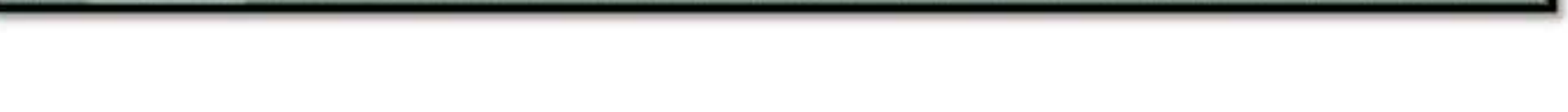

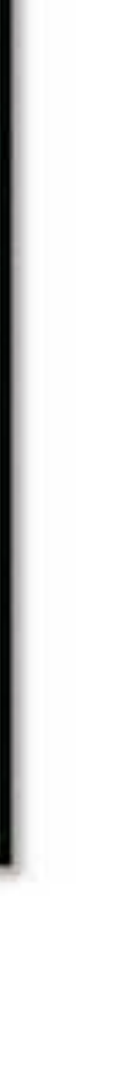


Sensor Locations

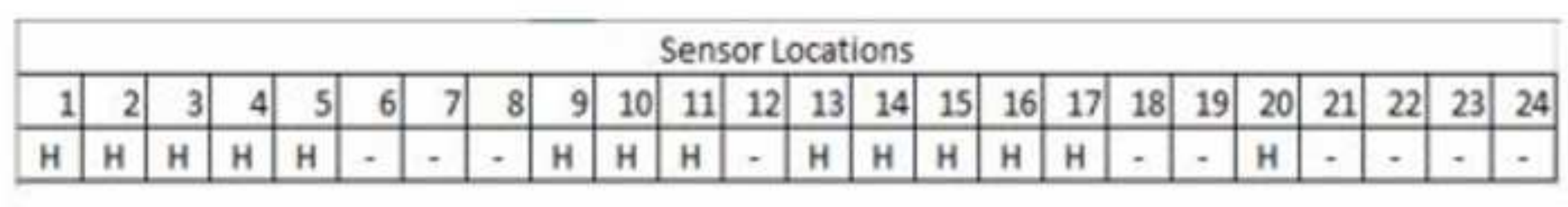 (r)}

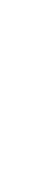

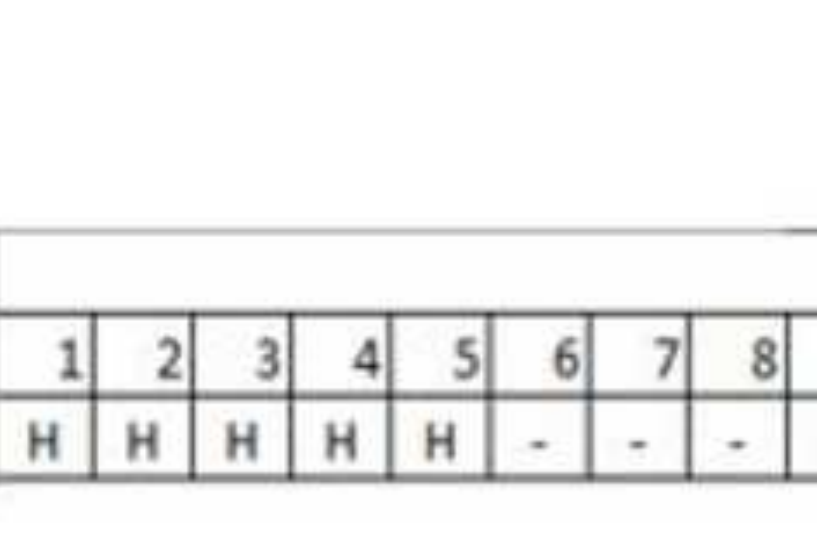

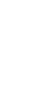

(

( ( 政 ( 


\begin{tabular}{|r|r|r|r|r|r|r|r|r|r|r|r|r|r|r|r|r|r|r|r|r|r|r|r|}
\hline 1 & 2 & 3 & 4 & 5 & 6 & 7 & 8 & 9 & 10 & 11 & 12 & 13 & 14 & 15 & 16 & 17 & 18 & 19 & 20 & 21 & 22 & 23 & 241 \\
\hline $\mathrm{H}$ & $\mathrm{M}$ & $\mathrm{L}$ & $\mathrm{M}$ & $\mathrm{H}$ & $\mathrm{M}$ & $\mathrm{L}$ & $\mathrm{L}$ & $\mathrm{L}$ & $\mathrm{L}$ & $\mathrm{L}$ & & $\mathrm{H}$ & $\mathrm{H}$ & $\mathrm{H}$ & $\mathrm{H}$ & $\mathrm{H}$ & $\mathrm{L}$ & - & $\mathrm{H}$ & $\mathrm{L}$ & $\mathrm{L}$ & $\mathrm{L}$ & $=$ \\
\hline
\end{tabular}




\begin{tabular}{|ll|}
\hline $\mathbf{Y}_{\mathbf{i}}$ & Description \\
\hline $\mathbf{1}$ & Gasifier syngas flow rate \\
\hline $\mathbf{2}$ & Syngas CO flow rate \\
\hline $\mathbf{3}$ & Syngas $\mathrm{CO}_{2}$ flow rate \\
\hline $\mathbf{4}$ & Syngas temperature \\
\hline $\mathbf{5}$ & Syngas pressure \\
\hline $\mathbf{6}$ & Low pressure steam turbine temperature \\
\hline $\mathbf{7}$ & Gas turbine combustor burn temperature \\
\hline $\mathbf{8}$ & Gas turbine combustor exit temperature \\
\hline $\mathbf{9}$ & Gas turbine high pressure exhaust stream temperature \\
\hline $\mathbf{1 0}$ & Gas turbine low pressure exhaust stream temperature \\
\hline $\mathbf{1 1}$ & Gas turbine expander output temperature \\
\hline $\mathbf{1 2}$ & Fluegas flow rate exiting gas turbine expander \\
\hline $\mathbf{1 3}$ & Syngas flow rate after solids removal \\
\hline $\mathbf{1 4}$ & Coal slurry flow rate entering gasifier \\
\hline $\mathbf{1 5}$ & Oxygen flow rate into gasifier \\
\hline $\mathbf{1 6}$ & Oxygen flow rate exiting ASU \\
\hline $\mathbf{1 7}$ & Acidgas flow rate \\
\hline $\mathbf{1 8}$ & Gas turbine compressor leakage flow rate \\
\hline $\mathbf{1 9}$ & Flow rate into high pressure steam turbine \\
\hline 20 & Coal slurry feed flow rate \\
\hline $\mathbf{2 1}$ & Slag extracted from syngas \\
\hline $\mathbf{2 2}$ & Fines extracted from syngas \\
\hline $\mathbf{2 3}$ & Gasifier heat output \\
\hline $\mathbf{2 4}$ & Recycled HRSG steam heat output \\
\hline
\end{tabular}

